\title{
Dinâmica e Teoria Cinética de Estados \\ Homogêneos de Sistemas com Interações de Longo Alcance
}

Christine Rebouças Lourenço

Tese apresentada ao Instituto de Física da Universidade

de Brasília como parte dos requisitos necessários para obtenção

do título de Doutora em Física.

Orientador: Prof. Dr. Tarcísio Marciano da Rocha Filho

Instituto de Física - Universidade de Brasília

10 de Agosto de 2016 


\section{Sumário}

1 Introdução 18

1.1 Interações de Longo-alcance . . . . . . . . . . . . . . . . . 18

1.2 Propriedades dos Sistemas de Interação de Longo-alcance . . . 19

1.2.1 Não-aditividade e Extensividade . . . . . . . . . . . 20

1.2.2 Inequivalência de Ensembles Estatísticos . . . . . . . 21

1.2.3 O Teorema do Virial . . . . . . . . . . . . 22

1.2.4 Calor Específico Negativo . . . . . . . . . . . . . . . 24

1.2.5 Quebra de Ergodicidade e Mixing . . . . . . . . . . . 26

1.3 O Espaço de Fase . . . . . . . . . . . . . . . . . 27

1.3.1 Equação de Liouville . . . . . . . . . . . . . . . . . 29

1.4 Relaxação Violenta em Sistemas com Interações de Longoalcance ....................... . . . . . . . . . . .

1.5 Estado Quasi-estacionário - QSS . . . . . . . . . . 32

1.5.1 Equação de Vlasov . . . . . . . . . . . . . . . 33

1.6 Equilíbrio Termodinâmico . . . . . . . . . . . . 36 
1.7 Dinâmica Rumo ao Equilíbrio no Modelo de Hertel-Thirring - Generalizado . . . . . . . . . . . . . . . 38

1.7.1 Resultados Inspiradores . . . . . . . . . . . 39

2 O Modelo Anisotrópico de Campo Médio 45

2.1 Apresentação do Modelo . . . . . . . . . . . . . . . . . 45

2.1.1 O Equilíbrio . . . . . . . . . . . . . 46

2.1.2 Relaxação Rumo ao Equilíbrio . . . . . . . . . . . . 51

2.2 O Estado Quasi-estacionário . . . . . . . . . . . 53

2.2.1 Os Efeitos de N Finito . . . . . . . . . . . . . 54

3 Teoria Cinética $\quad 61$

3.1 A Hierarquia BBGKY . . . . . . . . . . . . . . 62

3.2 Equação de Boltzmann . . . . . . . . . . . . . . . . . 67

3.3 Equação de Landau . . . . . . . . . . . . . . . . . . . . . 71

3.4 Equação de Balescu-Lenard . . . . . . . . . . . . . . 75

4 Dinâmica de Estados Homogêneos $\quad 76$

4.1 O Modelo Hamiltoniano de Campo Médio . . . . . . . . . . . 84

4.1.1 A Markovianização para o HMF . . . . . . . . . . 87

4.2 O Modelo do Anel . . . . . . . . . . . . . . . . . 91

4.2.1 A Markovianização para o Modelo do Anel . . . . . . . 92

5 Conclusões e Perspectivas $\quad 96$ 


\section{Agradecimentos}

Antes de tudo, agradeço a Deus.

Aos meus pais pelo exemplo, pelo apoio e pela motivação.

Aos meus irmãos por serem meu mais firme alicerce.

Ao Diego, amor da minha vida, por estar sempre ao meu lado.

Agradeço o apoio financeiro da CAPES.

Por fim, agradeço a meu orientador, pela paciência, por acreditar em mim e pela inspiração ao longo de todos esses anos. 
Ainda que tivesse o dom da profecia e conhecesse todos os mistérios e toda a ciência, ainda que tivesse toda a fé, de maneira tal que transportasse os montes, se não tivesse amor, nada seria.

Corintios 13:2 


\section{Resumo}

Sistemas autogravitantes, plasmas não-neutros, modelo do anel e Hamiltoniana de Campo Médio (HMF) são exemplos de sistemas e modelos com interações de longo-alcance que têm atraído muita curiosidade. Uma interação é dita de longo-alcance se ela decai na proporção $r^{-\alpha} \operatorname{com} \alpha \leq d$, em que $r$ é a distância de interação entre as partículas e $d$ é a dimensão espacial do sistema. Esses sistemas apresentam comportamentos não observados para sistemas com interações de curto-alcance, como calor específico negativo, inequivalência de ensembles, estados quasi-estacionários não-Gaussianos e relaxação violenta (uma passagem muito rápida da condição inicial para um estado quasi-estacionário, para só então evoluir muito lentamente para o equilíbrio termodinâmico ou oscilar em torno dele). Neste trabalho, vamos avaliar os sistemas com interação de longo-alcance começando pela forma como sua dinâmica evolui para o equilíbrio passando pela etapa de Relaxação Violenta; qual a relação entre o tempo de permanência no estado quasi-estacionário e o número de partículas dos sistemas e qual a influência das correlações de força na potência de $N$ que caracteriza essa dependência; além do verdadeiro 
equilíbrio termodinâmico por meio da maximização da entropia. 


\section{Abstract}

Self-gravitating systems, non-neutral plasmas, Ring-model and Hamiltonian Mean Field (HMF) are examples of systems and models with long-range interactions that have attracted much curiosity. An interaction is considered to be long-ranged if it decays at large distances as $r^{-\alpha}$ with $\alpha \leq d$ where $r$ is the distance between particles and $d$ is the spatial dimension of the system. These systems exhibit behavior not observed for systems with short range interactions, as negative specific heat, ensembles inequivalence, non-Gaussian quasi-stationary states and, especially, violent relaxation (a very rapid passage from the initial condition into a quasi-stationary state, which then evolves very slowly to the thermodynamic equilibrium or oscillates around it). In this work, we investigate the long-range interaction systems starting with the way its dynamics evolves towards thermodynamic equilibrium, passing by the violent relaxation stage; what is the relation between the long it takes in the quasi-stationary state and the number of particle systems and the influence of the correlations of force in the power of $N$ that characterizes this dependency; ending with a study of the thermodynamic equilibrium itself by 
an entropy maximization method. 


\section{Lista de Figuras}

1.1 Quarto Momento $\left\langle M_{4}\right\rangle$ da velocidade como função do tempo para o modelo da Hamiltoniana da equação (1.25) com uma condição inicial não-homogênea e energia por partícula 0.71. A simulação foi feita para $N=4000$ até $N=100000$ partículas e está sem reescala do tempo. . . . . . . . . . . . . . . 40

1.2 Quarto e sexto momentos $\left\langle M_{4}\right\rangle$ e $\left\langle M_{6}\right\rangle$ da velocidade como função do tempo para o modelo da Hamiltoniana da equação (1.25) com uma condição inicial não-homogênea e energia por partícula 0.71. A simulação foi feita para $N=4000$ até $N=$ 100000. No primeiro e no segundo quadros uma reescala com o fator $1 / N$ utilizada. . . . . . . . . . . . . . 41

1.3 Quarto Momento $\left\langle M_{4}\right\rangle$ da velocidade como função do tempo para o modelo da hamiltonina da equação (1.25) com uma condição inicial homogênea e energia por partícula 0.71. A simulação foi feita para $N=4000$ até $N=100000$. . . . . . 42 
1.4 Quarto Momento $\left\langle M_{4}\right\rangle$ da velocidade como função do tempo para o modelo da Hamiltoniana da equação (1.25) com uma condição inicial homogênea e energia por partícula 0.71. A simulação foi feita para $N=4000$ até $N=100000$. No primeiro e no segundo quadros a reescala foi feita proporcional a $1 / N^{2}$ e $1 / N^{1.7}$, respectivamente. . . . . . . . . . . . . 43

1.5 Sexto Momento $\left\langle M_{6}\right\rangle$ da velocidade como função do tempo para o modelo da Hamiltoniana da equação (1.25) com uma condição inicial homogênea e energia por partícula 0.71. A simulação foi feita para $N=4000$ até $N=100000$. No primeiro e no segundo quadros a reescala foi feita proporcional a $1 / N^{2}$ e $1 / N^{1.7}$, respectivamente. . . . . . . . . . . . 44

2.1 Quarto momento estatístico $\left\langle M_{4}\right\rangle$ da variável $\theta$ para $N=$ 10000 até $N=100000$ de um sistema homogêneo com energia $e=0.24$ sem reescala do tempo. . . . . . . . . . . . . 54

2.2 Momento $\left\langle M_{2}\right\rangle$ da variável $\theta_{i}$ como função do tempo para $N=$ 100000 do sistema homogêneo com energia $e=0,24 \ldots \ldots 56$

2.3 Momento $\left\langle M_{4}\right\rangle$ da variável $\theta_{i}$ como função do tempo para diferentes números de partículas $N=10000$ até $N=100000$ com reescala na forma $N^{-1,7}$ do sistema homogêneo com ener-

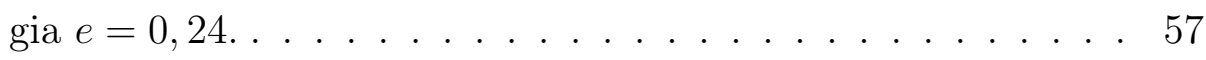


2.4 O mesmo que na Fig. (2.3) mas com a reescala do tempo na forma $N^{-2}$ do sistema homogêneo com energia $e=0,24 \ldots \quad . \quad 57$

2.5 O mesmo que na Fig. (2.3) mas com a reescala do tempo na forma $N^{-2} \ldots \ldots \ldots \ldots \ldots \ldots \ldots \ldots$

4.1 Autocorrelação de força $\mathcal{C}(t)$ como função do tempo para diferentes valores de $N$. As condições iniciais são as mesmas do estado homogêneo da Fig. 2.3 termalizada em $t=100.0$ antes de começar as simulações apresentadas. . . . . . . . . . . . . 82

4.2 Evolução temporal do quarto momento estatístico $\left\langle M_{4}\right\rangle$ da variável $\theta$ média sobre 1000 realizações exceto para $N=100000$ e $N=1000000$ com 300 e 200 realizações, respectivamente. As condições iniciais são as mesmas do estado homogêneo da Fig. 2.3 termalizada em $t=100.0$ antes de começar as simulações apresentadas. . . . . . . . . . . . . . . . . .

4.3 Evolução temporal da autocorrelação de força $\mathcal{C}(t)$. Média sobre 1000 realizações para $N=4000$ até $N=80000$ e 300 realizações para $N=100000$ partículas, utilizando uma condição inicial de water bag e e=0,69. . . . . . . . . . . 88

4.4 Evolução temporal do quarto momento estatístico $\left\langle M_{4}\right\rangle$ para a variável $\theta$. Média sobre 1000 realizaçõespara $N=4000$ até $N=80000$ e 300 realizações para $N=100000$ partículas, utilizando uma condição inicial de water bag e e=0,69. . . . . 89 
4.5 Evolução temporal da autocorrelação de força $\mathcal{C}(t)$. Média sobre 1000 realizações para $N=4000$ até $N=80000$ e 300 realizações para $N=100000$ partículas, utilizando uma condição inicial de water bag e e=0,8 . . . . . . . . . . . . . . . 90

4.6 Evolução temporal do quarto momento estatístico $\left\langle M_{4}\right\rangle$ para a variável $\theta$. Média sobre 1000 realizações para $N=4000$ até $N=80000$ e 300 realizações para $N=100000$ partículas, utilizando uma condição inicial de water bag e e=0,80. . . . . 91

4.7 $\theta_{i}$ e $\theta_{j}$ estão compreendidos no intervalo $-\pi<\theta<\pi$ e representam as posições angulares ocupadas pela i-ésima partícula no anel. . . . . . . . . . . . . . . . .

4.8 Evolução temporal do quarto momento estatístico $\left\langle M_{4}\right\rangle$ para a variável $\theta$. Média sobre 50 realizações para $N=4000$ até $N=60000$ e 5 realizações para os demais números de partículas, utilizando uma condição inicial de waterbag e $e=0,34$ termalizado em 100. . . . . . . . . . . . . . . . 93

4.9 Evolução temporal da autocorrelação de força $\mathcal{C}(t)$. Média sobre 50 realizações para $N=4000$ até $N=60000$ e 5 realizações para os demais números de partículas, utilizando uma condição inicial de waterbag e $e=0,34$ termalizado em 100. . 94 


\section{Lista de Tabelas}

2.1 Melhor reescala em $N^{\delta}$ para o momento $M_{4}$ entre um par de dados simulados com $N_{1}$ e $N_{2}$ partículas. . . . . . . . . . 59

4.1 Melhor reescala em $N^{\delta}$ para o momento $M_{4}$ entre um par de dados simulados com $N_{1}$ e $N_{2}$ partículas com energia $e=0,69 . \quad 89$

4.2 Melhor reescala em $N^{\delta}$ para o momento $M_{4}$ entre um par de dados simulados com $N_{1}$ e $N_{2}$ partículas com energia $e=0,8 . \quad 90$

4.3 Melhor reescala em $N^{\delta}$ para o momento $M_{4}$ entre um par de dados simulados com $N_{1}$ e $N_{2}$ partículas sem termalização. . . 94 


\section{Visão Geral}

Sistemas com interações de longo-alcance são aqueles em que o potencial de interação entre dois corpos decai com a distância de acordo com a relação $1 / r^{\alpha}$, onde $\alpha \leq d$, sendo $d$ a dimensão espacial do sistema. É o caso de sistemas gravitacionais, eletrostáticos e de hidrodinâmica bidimensional, por exemplo [12]. Neste trabalho, avaliaremos sistemas como o HMF, Modelo do Anel e um sistema de interação entre spins [30] bem como sua evolução dinâmica rumo ao equilíbrio (que também pode ser analisado sob a óptica das interações de longo-alcance).

Algumas propriedades destes sistemas têm despertado bastante interesse por violarem previsões básicas da Termodinâmica de curto-alcance. Elas frequentemente se originam da inequivalência de ensembles apresentada por sistemas com interação de longo-alcance.

Por muito tempo, características peculiares desses sistemas, como calor específico negativo e não-aditividade (embora não haja impedimento para que sejam extensivos), trouxeram dúvidas sobre a existência de um equilíbrio estatístico padrão nestes casos. No entanto, hoje sabemos que a evolução 
dinâmica leva ao equilíbrio após vencidas as etapas de relaxação violenta e estados quasi-estacionários, cuja duração é influenciada pelo número de partículas presentes. A compreensão da evolução temporal de uma dinâmica tão não-usual, é obtida pela introdução de uma teoria cinética apropriada baseada na equação de Vlasov [24, 27, 26].

O desenvolvimento computacional está bastante relacionado a grandes descobertas associadas à evolução dinâmica desses sistemas [21, 25]. A possibilidade de se efetuar simulações com números cada vez maiores de partículas em tempos razoáveis, além de nos levar a resultados mais fidedignos (na medida em que o número de partículas se aproxima do infinito, temos mais segurança nos resultados obtidos pela equação de Vlasov), nos permite ampliar o horizonte de análises e confrontar resultados anteriores com mais poder argumentativo. Neste trabalho, questionaremos a literatura anterior no que diz respeito ao expoente de proporcionalidade entre o número de partículas presentes em alguns sistemas e o seus tempos de evolução dinâmica. Um código paralelizado desenvolvido em nosso grupo para esse tipo de simulação é bastante utilizado.

O trabalho se inicia no capítulo 1 com uma breve revisão teórica sobre sistemas com comportamento de longo-alcance. Também é no capítulo 1 que apresento a motivação de meu estudo, ou seja, os resultados que nortearam e inauguraram o restante do trabalho.

No capítulo 2, consideraremos um modelo anisotrópico de Heisenberg que consiste em spins com interações de campo médio evoluindo sob a dinâmica 
clássica de spins, cuja Hamiltoniana é da forma

$$
H=-\frac{J}{2 N} \sum_{i, j=1}^{N} \vec{S}_{i} \cdot \vec{S}_{j}+D \sum_{i=1}^{N} S_{i z}^{2}
$$

em que o primeiro termo, com $J>0$, descreve um campo médio de acoplamento ferromagnético e o último termo representa a energia dada a uma anisotropia local. Estudaremos o comportamento evolutivo desse sistema bem como o seu equilíbrio final. Nas sessões finais desse capítulo, apresentamos os resultados das simulações computacionais realizadas para o modelo de spins clássicos em que se evidencia a dependência entre o número de partículas no sistema e o tempo de permanência no estado quasi-estacionário (QSS). Um comparativo entre o expoente que relaciona essas duas variáveis para sistemas cada vez maiores é apresentado e, pela primeira vez, é feita uma discussão sobre o que justifica a discrepância entre os valores desse expoente encontrado em resultados de simulações computacionais e a previsão teórica [28] para pequenos números de partículas. Em outras palavras, justificamos o aparecimento de uma relação do tipo $1 / N^{1.7}$ como reescala do tempo dinâmico de simulações em que o número de partículas é limitado.

A Teoria Cinética, abordada ao longo de todo o trabalho, ganha um lugar especial no capítulo 3 em que é apresentada formalmente a Hierarquia Bogoliubov-Born-Green-Krikwood-Yvon (BBGKY) e algumas equações cinéticas são derivadas e analisadas.

O capítulo 4 traz discussões sobre a influência das correlações de força 
na ordem do termo predominante quando se analisam a dependência entre $N$ e o tempo da dinâmica para modelos teóricos bem conhecidos: o Modelo Hamiltoniano de Campo Médio (HMF), cuja Hamiltoniana tem a forma

$$
H=\sum_{i=1}^{N} \frac{p_{i}^{2}}{2}+\frac{1}{2 N} \sum_{i, j=1}^{N}\left[1-\cos \left(\theta_{i}-\theta_{j}\right)\right]
$$

em que $\theta_{i} \in\left[-\pi, \pi\left[\right.\right.$ e $p_{i}$ é o momento canonicamente conjugado à $\theta_{i}$ e o Modelo do Anel com Hamiltoniana escrita como

$$
H=\sum_{i=1}^{N} \frac{p_{i}^{2}}{2 m_{i}}+\frac{1}{2 N^{2}} \sum_{i<j=1}^{N} \frac{1}{\sqrt{1-\cos \left(\theta_{i}-\theta_{j}\right)+\varepsilon}}
$$

em que $\theta_{i}$ e $\theta_{j}$ estão compreendidos no intervalo $-\pi<\theta<\pi$ e representam as posições angulares ocupadas pela i-ésima partícula no anel e $\varepsilon$ é um parâmetro de amortecimento computacional.

Encerro este trabalho com o capítulo 5 em que apresento minhas conclusões e perspectivas para estudos futuros. 


\section{Capítulo 1}

\section{Introdução}

\subsection{Interações de Longo-alcance}

A análise de cenários bastante robustos evidencia grandes distinções entre os sistemas de interações de curto-alcance e os de interações de longo-alcance. Neste último caso, a dinâmica rumo ao equilíbrio possui características que ainda não foram satisfatoriamente estudadas, como a relaxação violenta e a existência de estados quasi-estacionários [12]. Assim, sistemas com interações de longo-alcance configuram um campo bastante intrigante e repleto de lacunas a serem preenchidas.

Quanto à evolução dinâmica citada acima, sabe-se que o sistema, a partir de uma condição incial qualquer, passa por uma etapa muito rápida chamada "Relaxação Violenta"(inicialmente descrita por Lynden-Bell [8]) para, só então, atingir um estado quasi-estacionário ou oscilar em torno dele. A 
descrição de Lynden-Bell [8], no entanto, não representava satisfatoriamente os resultados obtidos na Astrofísica, servindo como motivação para outros trabalhos, tais como Shu (1978), Stiavelli Bertin (1987), Spergel e Hernquist (1992), Kull, Treumann e Bohringer (1997), Nakamura (2000) e Trenti e Bertin (2002) e Yan Levin (2008). Apesar de interessantes resultados obtidos, nenhuma dessas teorias é de aplicação geral.

Partindo agora para a etapa quasi-estacionária, sabe-se que o tempo de vida desses estados é fortemente influenciado pela quantidade de partículas $(N)$ presentes no sistema, de forma que, no caso $N \rightarrow \infty$ o sistema permaneceria "preso" nesta etapa intermediária e o equilíbrio nunca seria atingido. Sistemas menores evoluem para o equilíbrio gaussiano em um tempo crescente na proporção $N$ ou $N^{2}$ dependendo de sua condição incial (fato que discutiremos neste trabalho).

Apresentamos algumas propriedades dos sistemas com interações de longoalcance:

\subsection{Propriedades dos Sistemas de Interação de Longo-alcance}

Neste capítulo, iremos discutir o que faz dos sistemas com interações de longo-alcance sistemas tão peculiares. 


\subsubsection{Não-aditividade e Extensividade}

Sistemas com interações de longo-alcance não são aditivos embora, em geral, sejam extensivos [16].

Extensividade é a propriedade que qualifica um sistema em que as variáveis termodinâmicas dependem de seu tamanho. Sistemas extensivos podem algumas vezes ser reconhecidos pela presença do fator de Kac em suas Hamiltonianas. Trata-se do fator $1 / N$ que torna a energia do sistema proporcional ao número de partículas. Essa reescala de energia e entropia constitui importante aspecto na transição de fase.

A aditividade, por sua vez, é a propriedade que se evidencia quando o sistema é dividido em dois subsistemas e a energia total $E$ é igual à simples adição das respectivas energias $E_{1}$ e $E_{2}$ de cada subsistema. Em sistemas com interação de curto-lcance, no limite termodinâmico, a característica da interação permite que se ignore o termo de interface. Para sistemas com interação de longo-alcance, se dividirmos o sistema em duas partes, a energia total do sistema não será igual à soma das energias dos dois subsistemas [16], ou seja, em sistemas com interação de longo-alcance, o termo de interface considera a totalidade de partículas. Para este caso, a interação através da interface não pode ser negligenciada. Sistemas com interação de longoalcance são não-aditivos.

Note que todo sistema aditivo é extensivo, mas nem todo sistema extensivo é aditivo, como é o caso dos sistemas que serão analisados no presente trabalho. 


\subsubsection{Inequivalência de Ensembles Estatísticos}

Diferentemente de sistemas com interações de curto-alcance, sistemas com interações de longo-alcance podem apresentar inequivalências entre os ensembles canônico e microcanônico. Alguns exemplos são as interações gravitacionais e o modelo BEG (Blume-Emery-Griffiths) [7][44].

O estudo de Barré (analisando diagramas de fase nos ensembles canônico e microcanônico) evidenciou que, no modelo BEG-spin 1, o ensemble canônico exibia um diagrama de fase com transição contínua no ponto crítico, enquanto no ensemble microcanônico o sistema possui calor específico negativo e um salto na temperatura.

O tipo de ensemble (canônico ou microcanônico) corresponde ao vínculo físico associado a ele ( temperatura ou energia constantes, respectivamente), características que provocam o aparecimento de diferentes propriedades físicas. Gibbs notou, em 1902, que, no limite termodinâmico, os dois ensembles levam a previsões que podem ser inequivalentes [29, 35]. Muitos outros trabalhos posteriores revelaram o mesmo comportamento em sistemas de muitos corpos $[35,36]$. Logo, a equivalência ou não dos dois ensembles depende do tipo de interação presente no sistema em análise.

A inequivalência de ensembles ocorre quando a função de entropia microcanônica por partícula para um sistema com $n$ partículas dada por:

$$
s(u)=\lim _{n \rightarrow \infty} \frac{1}{n} \ln \Omega(u),
$$


em que $\Omega(u)$ é a densidade de microestados com energia interna por partícula $u$, apresenta um ou mais intervalos não-côncavos que faz de $s^{\prime}$, a primeira derivada de $s(u)$, uma função não-monotônica de $u$. A energia livre é obtida como a transformada de Legendre da $s(u)$ :

$$
\varphi(\beta)=s(u)-T u=\lim _{n \rightarrow \infty}-\frac{1}{n} \ln Z(\beta),
$$

em que $Z(\beta)$ é a função-partição e $T=\left(\frac{\partial S}{\partial u}\right)^{-1}$, a temperatura. A transformada de Legendre de $s(u)$ não é inversível. Por outro lado, se $s^{\prime}$ existir para todo $u$ e for monotônica, $s$ é então a transformada de Legendre de $\varphi(\beta)$ :

$$
s(u)=\beta(u) u-\varphi(\beta(u)),
$$

com $\beta(u)=s^{\prime}(u)$, que corresponde à equivalência dos ensembles canônico e microcanônico.

Nos sistemas com interações de longo-alcance, os ensembles canônico e microcanônico podem não ser equivalentes. Essa característica deriva da não-aditividade oriunda da natureza da interação.

\subsubsection{O Teorema do Virial}

Alguns sistemas apresentam oscilações de densidade. Essas flutuações não são soluções estacionárias da equação de Vlasov durante o processo de relaxação e, para diminuí-las, pode-se utilizar uma configuração que minimiza o desbalanço entre energia cinética e potencial: a condição do virial. Em um 
estado estacionário, o virial $G=\langle\mathbf{p . q}\rangle$ não depende do tempo, portanto

$$
\frac{d}{d t}\langle\mathbf{p . q}\rangle=\left\langle\frac{d}{d t}(\mathbf{p . q})\right\rangle=0
$$

ou

$$
\left\langle p^{2}\right\rangle=-\left\langle\mathbf{q} \cdot\left(\frac{d}{d t} \dot{\mathbf{q}}\right)\right\rangle=0 .
$$

Considerando que a média temporal $(\langle\rangle)$ é equivalente à média sobre as partículas e utilizando as equações de Hamilton, podemos escrever

$$
\left\langle p^{2}\right\rangle=-\frac{1}{N} \sum_{i=1}^{N} \mathbf{F}_{i} \cdot \mathbf{q}_{i}
$$

Em termos da função distribuição reduzida a uma partícula (de que trataremos nas próximas seções) a eq. 1.4 pode ser reescrita como

$$
\left\langle p^{2}\right\rangle=-\int d \mathbf{q} d \mathbf{p} f(\mathbf{q}, \mathbf{p})\left[-\frac{\partial V(\mathbf{q})}{\partial \mathbf{q}} \cdot \mathbf{q}\right]
$$

em que $V(\mathbf{q})$ é o potencial de campo médio. Isso implica que, em um sistema de partículas com interações de longo-alcance, se a energia cinética média do sistema $\left\langle E_{c}\right\rangle$ e a energia potencial média do sistema $\left\langle E_{p o t}\right\rangle$ não variam significativamente no tempo, então

$$
\left\langle E_{c}\right\rangle=-\frac{1}{2}\left\langle E_{p o t}\right\rangle .
$$




\subsubsection{Calor Específico Negativo}

Em Astrofísica, o calor específico negativo, como consequência do Teorema do Virial, foi exaustivamente discutido por Maxwell [45], Thirring [59] e Lynden-Bell [42], entre outros. Sabe-se que, quando uma estrela ou um conjunto delas perde energia, sua temperatura aumenta de acordo com esse teorema. O mesmo acontece com buracos negros, como demonstrado por Beckestein e Hawking, em 1974 [32]. Esse fato é bastante intrigante e paradoxal sob o ponto de vista da Mecânica Estatística, pois, aparentemente, o calor específico deve ser sempre positivo. De fato, se consideramos um sistema extensivo uniforme e dividido em partes de modo que o calor possa fluir entre elas, ele será. A segunda lei da Termodinâmica afirma que o calor flui da parte mais quente para a mais fria, evidenciando um gradiente de temperatura. Se o calor específico for negativo o sentido do fluxo de calor se inverte e a diferença de temperatura irá sempre aumentar de forma que o sistema jamais atingiria o equilíbrio térmico. A positividade do calor especí-

fico é, assim, uma condição de estabilidade do sistema. É importante notar que esse argumento só é válido se o sistema for aditivo, o que não é o caso dos sistemas aqui considerados. Com efeito, se considerarmos um sistema descrito pelo ensemble canônico de Gibbs, sua energia interna, considerando níveis de energia $E_{i}$, é dada por

$$
\langle E\rangle=\frac{\sum_{i} E_{i} e^{-\beta E_{i}}}{Z}=-\frac{\partial \ln Z}{\partial \beta},
$$


sendo $Z$ a função-partição. O calor específico a volume constante é sempre positivo, pois

$$
C_{v}=\frac{\partial\langle E\rangle}{\partial T} \propto\left\langle(E-\langle E\rangle)^{2}\right\rangle>0 .
$$

Por outro lado, o teorema do virial para um sistema autogravitante nos dá [10], como em (1.5),

$$
2\left\langle E_{c}\right\rangle+\left\langle E_{\text {pot }}\right\rangle=0,
$$

em que $\left\langle E_{c}\right\rangle$ e $\left\langle E_{p o t}\right\rangle$ são as médias estatísticas das energia cinética e potencial, respectivamente, para uma distribuição de posições e velocidades estacionária. Temos então que:

$$
E=\left\langle E_{c}\right\rangle+\left\langle E_{p o t}\right\rangle=-\left\langle E_{c}\right\rangle,
$$

e portanto:

$$
C_{v}=\frac{\partial\langle E\rangle}{\partial T} \propto \frac{\partial\langle E\rangle}{\partial E_{c}}<0 .
$$

Apesar de (1.7) e (1.10) sugerirem uma contradição, salientamos que ela ocorre porque estamos aplicando a teoria a diferentes ensembles (o canônico e o microcanônico) com vínculos físicos distintos em cada caso.

Além do contexto astrofísico, há evidência experimental do calor específico negativo microcanônico para sistemas pequenos [10]. Um deles é a fragmentação nuclear, para a qual foram feitas medidas consecutivas da tem- 
peratura e da energia do ensemble durante a colisão entre átomos de $\mathrm{Au}$ [15].

\subsubsection{Quebra de Ergodicidade e Mixing}

A hipótese de ergodicidade de Boltzmann consiste em que um sistema em evolução livre por um tempo suficientemente longo passa por todos os estados compatíveis com suas condições gerais para um determinado valor de energia [4], [58], [20]. Isso pode ser interpretado como um grande sistema de partículas interagentes em equilíbrio em que a média temporal se aproxima da média do ensemble de partículas [54],[19].

A Hipótese Ergódica pode ser enunciada por:

- O tempo médio que um sistema passa numa região do espaço de fase é proporcional à medida da própria região;

- Existe uma, e somente uma, distribuição de probabilidades que é invariante no que diz respeito às transformações pertinentes ao sistema.

Como consequencia disso, temos que, num sistema ergódico, as médias temporais são iguais às médias microcanônicas, ou seja, ergodicidade é a propriedade que caracteriza um sistema em que a média de uma grandeza sobre uma única partícula é igual à média do ensemble. Assim, para que se entenda o comportamento dinâmico de um sistema ergódico como um todo, basta que estudemos o comportamento médio de uma de suas partículas 
[19]. Michaelis e colaboradores, em [19], observaram experimentalmente a ergodicidade.

Há ainda uma outra hipótese equivalente à hipótese ergódica à luz da Mecânica Estatística: a hipótese de mixing, de Gibbs [2]. Diferentemente de Boltzmann, Gibbs interpretou a evolução de um conjunto de partículas puntiformes que se dispersa como um fluido incompressível que, eventualmente, ocupará todo o espaço de fase (de que trataremos nas próximas sessões), caracterizando o estado de mixing. Gibbs argumentou que, se o mixing ocorrer, o comportamento médio desse conjunto de partículas será o comportamento verificado em laboratório. Isso significa dizer que a função de autocorrelação do sistema decai com o tempo. Na prática, as duas hipóteses são verificadas em sistemas com interações de curto-alcance. Por outro lado, para sistemas com interações de longo-alcance, devido à prescrição de Kac, acontece exatamente o oposto. Escalar o potencial com $1 / N$ aniquila as correlações entre as partículas e são justamente essas correlações que levam o sistema ao equilíbrio termodinâmico de acordo com Boltzmann.

Sistemas com interações de longo-alcance apresentam quebra de ergodicidade (não são ergódicos) e de mixing.

\subsection{O Espaço de Fase}

Para compreendermos a evolução temporal de um sistema com interações de longo-alcance, precisamos conhecer a Teoria Cinética que apresentamos 
de maneira resumida nesta seção [46] e que será abordada de maneira mais completa no capítulo 3 .

Define-se o espaço de fase $\Gamma$ de um sistema com $N$ graus de liberdade como sendo um espaço cartesiano de $2 N$ dimensões com coordenadas $\left\{q_{i}, p_{i}\right\}$ em que $q$ é a posição e $p$, o momento. A trajetória do sistema será uma curva em $\Gamma$ parametrizada pelo tempo $t$ :

$$
\left[q_{1}(t), \ldots, q_{N}(t) ; p_{1}(t), \ldots, p_{N}(t)\right]
$$

A Hamiltoniana genérica para um sistema formado por $N$ partículas de massas unitárias e um potencial de interação de pares $V(q)$ será

$$
\begin{aligned}
& H=\sum_{i=1}^{N} \frac{p_{i}^{2}}{2}+\frac{1}{2} \sum_{i<j=1}^{N} V\left(\left|q_{i}-q_{j}\right|\right), \\
& \dot{q}_{i}=\partial_{p_{i}} H \\
& \dot{p}_{i}=-\partial_{q_{i}} H=-\sum_{i<j=1}^{N} \partial_{q_{i}} V\left(\left|q_{i}-q_{j}\right|\right) .
\end{aligned}
$$

A função

$$
f_{N}\left(q_{1}, \ldots, q_{N}, p_{1}, \ldots, p_{N} ; t\right)\left(d^{N} q\right)^{d}\left(d^{N} p\right)^{d}
$$

é a função distribuição de probabilidades a $N$ partículas e representa a probabilidade de encontrarmos as $N$ partículas no elemento $\left(d^{d N} q\right)\left(d^{d N} p\right)$ com posições $q_{1}, \ldots, q_{d N}$ e momentos $p_{1}, \ldots, p_{d N}$, no instante de tempo $t$. 


\subsubsection{Equação de Liouville}

Considere $d N$ o número de partículas contidas em um elemento de volume $d V$ de $\Gamma$ [46]. Da definição de função densidade, podemos escrever

$$
f_{N}(p, q ; t) d q d p=f(q, p ; t) d V
$$

e, com isso,

$$
f_{N}(q, p ; t)=\frac{d N}{d V}
$$

Ou seja, $f(q, p ; t)$ representa a densidade de partículas por unidade de volume $d V \in \Gamma$. Podemos ainda reduzir a função distribuição anterior para um subgrupo formado por $s$ partículas da seguinte maneira

$$
f_{s}(1 \ldots s) \equiv \int d(s+1) \ldots d(N) f_{N}(1 \ldots s(s+1) \ldots N)
$$

em que utilizamos a simplificação de notação a seguir

$$
\left(x_{1} \ldots x_{N}, v_{1} \ldots v_{N}\right) \equiv(1 \ldots N) \text {. }
$$

Considerando apenas sistemas conservativos e sem colisões, cada partícula do sistema forma um subsistema e a função distribuição $f(q, p ; t)$ obedece ao teorema de Liouville

$$
\frac{d f_{N}}{d t}=0
$$


e

$$
\partial_{t} f_{N}+\sum_{i=1}^{N}\left\{\partial_{q_{i}} f_{N} \frac{d q_{i}}{d t}+\partial_{p_{i}} f_{N} \frac{d p_{i}}{d t}\right\}=0
$$

em que a notação $\left\{q_{i}, p_{i}\right\}=\left\{q_{1}, \ldots, q_{N}, p_{1}, \ldots, p_{N}\right\}$ foi adotada. Reescrevendo a equação anterior levando-se em consideração as equações de Hamilton, temos:

$$
\partial_{t} f_{N}+\sum_{i=1}^{N}\left\{\partial_{q_{i}} f_{N} \partial_{p_{i}} H-\partial_{p_{i}} f_{N} \partial_{q_{i}} H\right\}=0 .
$$

A equação anterior é a Equação de Liouville.

Ao se integrar a função distribuição reduzida (f.d.r.) a $s$ partículas $f_{s}$ obtém-se um sistema de tamanho proporcional a $N$ de equações (uma hierarquia) em que $f_{s}$ depende de $f_{s+1}$ :

$$
\begin{aligned}
\partial_{t} f_{s}(1, \ldots, s) & +\sum_{j=1}^{s} \mathbf{v}_{j} \cdot \nabla_{j} f_{s} \\
& =\left(\lambda^{2} / m\right) \sum_{j=1}^{s} \int d \mathbf{x}_{s+1} d \mathbf{v}_{s+1}\left(\nabla_{j} V_{j, s+1}\right) \cdot \partial f_{s+1}(1, \ldots, s+1),
\end{aligned}
$$

conhecida como hierarquia BBGKY (desenvolvida de forma por Bogoliubov, Born, Green, Krikwood e Yvon), de que trataremos ao longo do trabalho $[55]$. 


\subsection{Relaxação Violenta em Sistemas com Inte- rações de Longo-alcance}

As partículas de um sistema (com $N$ grande e fora do equilíbrio), trocando forças de longo-alcance, oscilam inicialmente com amplitude decrescente a fim de atingir um estado quasi-estacionário. O tempo necessário para alcançar esse estado foi estimado por Lynden-Bell como sendo da ordem de algumas oscilações (ou seja, bastante curto) em sistemas estelares, justificando a expressão "relaxação violenta". Segundo ele, numa galáxia em que o campo gravitacional médio não é estável e que relaxa para o quasi-equilíbrio, o tempo de relaxação violenta pode ser escrito na forma

$$
T_{r} \approx \frac{3 P *}{8 \pi}
$$

em que $P *=2 \pi / n$ é a frequência de vibração da galáxia e:

$$
n=(2 \pi G \bar{\rho})^{-1 / 2}
$$

$\operatorname{com} \bar{\rho}$ representando a densidade média dada por $\bar{\rho}=M /\left(\frac{4}{3} \pi R_{0}^{3}\right)$.

Embora seja uma referência inicial para o tema, o trabalho de LyndenBell ainda apresentava falhas. Outros trabalhos surgiram a fim de sanar estes problemas, como os que se baseiam em uma aproximação mais fundamental da dinâmica dos sistemas, partindo de uma maximização da entropia $[53,57$, 33, 37, 9]. Essa é a abordagem utilizada neste trabalho. 
Todas essas teorias têm como objetivo predizer o estado final após a relaxação violenta a partir do estado inicial. Um problema apresentado por todas as abordagens estudadas é a natureza não-transitiva, mostrada por Arad e Lynden-Bell [3]. Eles consideram um sistema gravitante em um estado fora do equilíbrio, com energia inicial $E_{1}$, que relaxa até um primeiro estado $s_{1}$. O sistema é então perturbado por uma força externa que induz uma variação de energia $\Delta E$ e, por ocasião desta perturbação, sofre uma nova relaxação violenta, desta vez para um estado $s_{2}$ com energia $E_{2}$. Caso tomássemos o mesmo sistema, com as mesmas condições, a despeito da energia inicial que agora seria $E+\Delta E$, ele relaxaria apenas uma vez e para um estado $s_{3}$, diferente de $s_{2}$.

A não-transitividade é sinal de que a descrição cinética da relaxação violenta é, provavelmente, incompleta já que o equilíbrio é independente do caminho de evolução do sistema. Ainda é necessária uma abordagem dinâmica para encontrar a teoria apropriada para determinar a função grão-grosso da densidade do espaço de fase.

\subsection{Estado Quasi-estacionário - QSS}

Estados quasi-estacionários (QSS, do inglês, "quasi-stationary states") são estados dinâmicos nos quais o sistema apresenta algumas características semelhantes às do equilíbrio, no entanto não correspondem ao equilíbrio previsto pela mecânica estatística em virtude de alguns aspectos, por exem- 
plo o fato de que as quantidades macroscópicas do sistema não obedecem às previsões da Mecânica Estatística. No QSS, uma variável macroscópica do sistema pode sofrer, eventualmente, uma alteração que aconteceria em um tempo que aumenta com o crescimento do número de partículas envolvidas.

Existem alguns trabalhos com simulações numéricas que apresentam essas informações [14]. A duração temporal do estado quasi-estacionário pode ser bem longa e por isso é importante compreender os aspectos desse estado. A equação de Vlasov é uma ferramenta importante para essa compreensão, uma vez que possibilita uma descrição teórica dos estados quasi-estacionários e o elemento temporal é discutido e analisado em comparação com simulações numéricas. Deste modo, Lynden-Bell [8] desenvolveu sua teoria de relaxação violenta, em que utiliza a equação de Vlasov, cujas características possibilitam prever o estado quasi-estacionário para o modelo HMF.

\subsubsection{Equação de Vlasov}

Para descrever o estado de um sistema, pode-se projetar todas as posições e momentos no espaço de fase e/ou utilizar uma distribuição de $N$ partículas associadas ao espaço de fase da distribuição [51]. A distribuição será representada por $f_{N}(\mathbf{Q}, \mathbf{P}, \mathbf{t})$, em que $(\mathbf{Q}, \mathbf{P}, \mathbf{t})$ representa o conjunto completo de todas as posições e momentos $\left(q_{i}, p_{i}\right)$ ao longo do tempo. A cada instante, a probabilidade do sistema de $N$ partículas apresentar posições e momentos em uma região $d \mathbf{Q} d \mathbf{P}$ em torno de $(\mathbf{Q}, \mathbf{P})$ pode ser calculada por $f_{N}(\mathbf{Q}, \mathbf{P}) d \mathbf{Q} d \mathbf{P}$ e a evolução da função $f_{N}$ é governada pela equação de Liouville descrita pelo 
parêntese de Poison

$$
\{A, B\}=\sum_{i=1}^{N}\left(\frac{\partial A}{\partial q_{i}} \frac{\partial B}{\partial p_{i}}-\frac{\partial A}{\partial p_{i}} \frac{\partial B}{\partial q_{i}}\right) .
$$

Frequentemente, apenas nos interessa a evolução temporal de uma única partícula. Nesse caso, é mais conveniente utilizar a função distribuição reduzida a $s$ partículas definida por

$f_{s}\left(\mathbf{q}_{1}, \mathbf{p}_{1}, \ldots, \mathbf{q}_{s}, \mathbf{p}_{s} ; t\right)=\frac{N !}{(N-s) !} \int d \mathbf{p}_{s+1} d \mathbf{q}_{s+1} \cdots d \mathbf{q}_{N} d \mathbf{p}_{N} f_{N}\left(\mathbf{q}_{1}, \mathbf{p}_{1}, \ldots, \mathbf{q}_{N}, \mathbf{p}_{N} ; t\right)$

e fazer $s=1$.

Há dois casos relevantes para a função distribuição reduzida: as partículas são estatisticamente independentes ou as partículas são estatisticamente correlacionadas. No primeiro caso, podemos escrever a função distribuição reduzida a $s$ partículas pelo produtório a seguir

$$
f_{s}\left(\mathbf{q}_{1}, \mathbf{p}_{1}, \ldots, \mathbf{q}_{s}, \mathbf{p}_{s} ; t\right)=C \prod_{j=1}^{s} f_{1}\left(\mathbf{q}_{j}, \mathbf{p}_{j} ; t\right)
$$

em que $C$ é uma constante de proporcionalidade. Quando existe correlação entre partículas, a função distribuição reduzida deve ser escrita na forma de expansão em aglomerados que deve ser compatível com o sistema em estudo.

A evolução temporal da função distribuição a uma partícula será obtida da integração da equação de Liouville em todo o espaço de fase utilizando-se a aproximação conveniente para o tipo de correlação do sistema na função 
distribuição reduzida. No caso de um sistema que apresente correlações a duas partículas, por exemplo, teremos a função distribuição reduzida a uma partícula escrita como

$$
f_{1}\left(\left(\mathbf{q}_{1}, \mathbf{p}_{1}\right),\left(\mathbf{q}_{2}, \mathbf{p}_{2}\right)\right)=f_{1}\left(\mathbf{q}_{1}, \mathbf{p}_{1}\right) f_{1}\left(\mathbf{q}_{2}, \mathbf{p}_{2}\right)+C_{2}\left(\left(\mathbf{q}_{1}, \mathbf{p}_{1}\right),\left(\mathbf{q}_{2}, \mathbf{p}_{2}\right)\right),
$$

em que $C_{2}$ é a correlação a duas partículas e a dependência temporal está implícita. A integração da respectiva equação de Liouville nos dá

$$
\begin{aligned}
\frac{\partial}{\partial t} f_{1}\left(\mathbf{q}_{1}, \mathbf{p}_{1}\right) & +\mathbf{p}_{1} \cdot \frac{\partial}{\partial \mathbf{q}_{1}} f_{1}\left(\mathbf{q}_{1}, \mathbf{p}_{1}\right)=\int d \mathbf{q}_{2} d \mathbf{p}_{2} \frac{\partial}{\partial \mathbf{q}_{1}} V\left(\left|\mathbf{q}_{1}-\mathbf{q}_{2}\right|\right) \frac{\partial}{\partial \mathbf{p}_{1}} f_{1}\left(\mathbf{q}_{1}, \mathbf{p}_{1}\right) f_{1}\left(\mathbf{q}_{2}, \mathbf{p}_{2}\right) \\
& +\int d \mathbf{q}_{2} d \mathbf{p}_{2} \frac{\partial}{\partial \mathbf{q}_{1}} V\left(\left|\mathbf{q}_{1}-\mathbf{q}_{2}\right|\right)\left(\frac{\partial}{\partial \mathbf{p}_{1}}-\frac{\partial}{\partial \mathbf{p}_{2}}\right) C_{2}\left(\left(\mathbf{q}_{1}, \mathbf{p}_{1}\right),\left(\mathbf{q}_{2}, \mathbf{p}_{2}\right)\right) .
\end{aligned}
$$

Na equação acima, o primeiro termo à direita é o termo de Vlasov, responsável pela aceleração das partículas. Note que este termo carrega uma média do potencial no espaço de fase. O próximo termo, também à direita é o termo que carrega a correlação entre as partículas.

Sobre a ordem de grandeza dos termos acima, em sistemas com interação de longo-alcance, podemos supor que o potencial de interação é fraco e, portanto, de ordem $\lambda(V(r)=O(\lambda))$. Assim, supondo $\lambda$ um parâmetro muito pequeno e mantendo apenas os termos de ordem $\lambda^{1}$ para um sistema espacialmente unidimensional e considerando-se as coordenadas cartesianas, 
tem-se a equação de Vlasov na forma

$$
\frac{\partial f}{\partial t}+p \frac{\partial f}{\partial x}+F[f, V] \frac{\partial f}{\partial p}=0
$$

em que

$$
F[f, V]=\int d x^{\prime} d p^{\prime} f\left(x^{\prime}, p^{\prime}\right) \frac{d}{d x} V\left(\left|x-x^{\prime}\right|\right)
$$

é a força média.

\subsection{Equilíbrio Termodinâmico}

No ensemble canônico, o equilíbrio termodinâmico é caracterizado pela mínima Energia Livre de Helmholtz $F(N, V)=U(N, V)-T S(N, V)$, com $S(N, V)$ sendo a entropia do sistema (dada pela equação de Boltzmann $S=$ $k_{B} \ln W$, em que $W$ é o número de microestados disponíveis do sistema) e $T$, a temperatura do banho térmico.

O volume do espaço de fase, para sistemas hamiltonianos é proporcional a $W$, portanto $S \sim N$ independente do tipo de interação entre as partículas do sistema. Assim, para sistemas com interações de curto-alcance, tanto a energia quanto a entropia escalam linearmente com o número de partículas, mas, para sistemas com interação de longo-alcance, a energia interna é da forma $U \sim N^{2}$ e o mínimo da energia Livre de Helmholtz será dado pelo mínimo da energia interna, a menos que a temperatura do banho escale linearmente com o número de partículas, resultando numa termodinâmica 
trivial. Nestes casos, deve-se reescalar a energia por um fator $1 / N$ atendendo a prescrição de Kac [2].

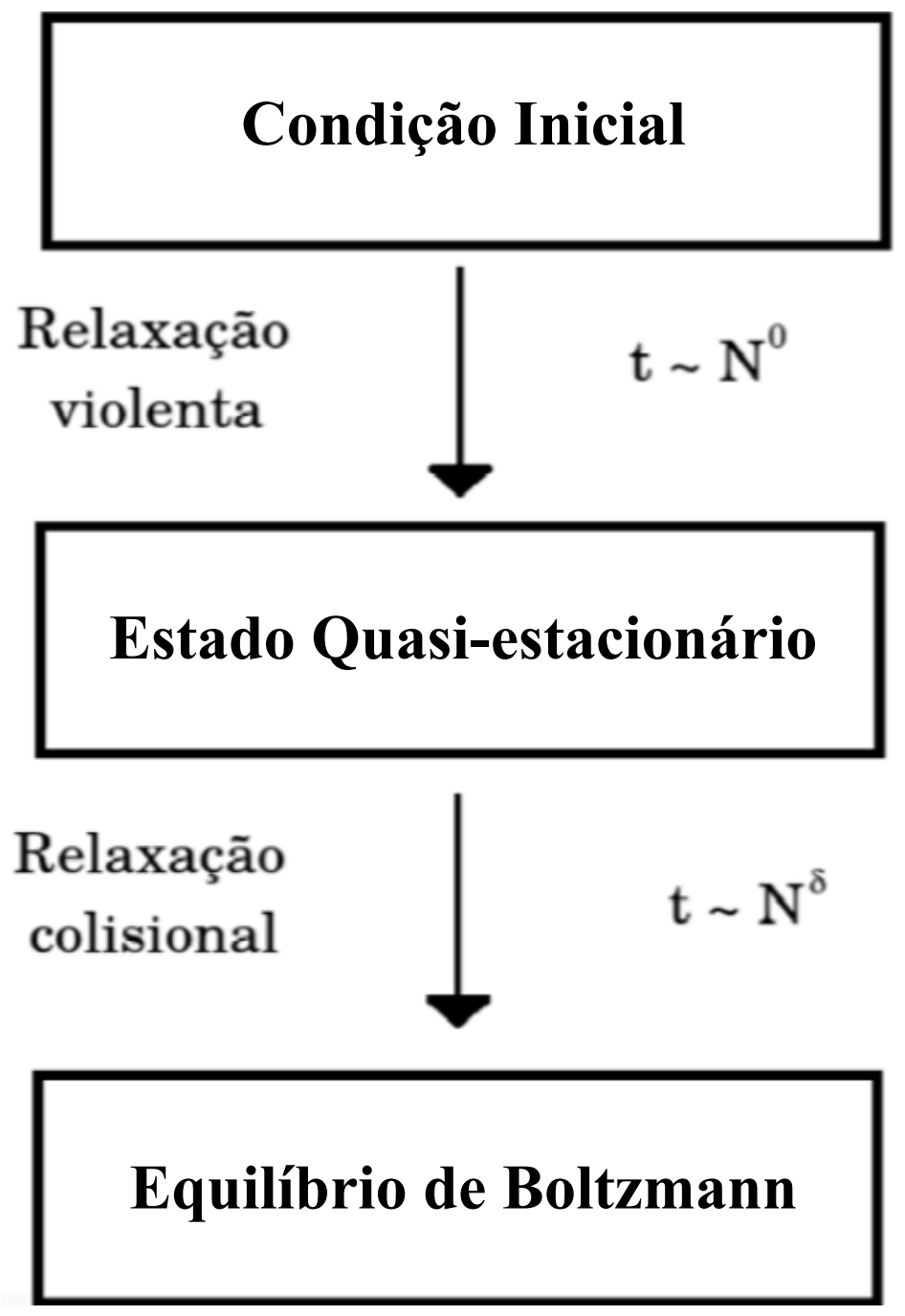

Estatisticamente, o equilíbrio termodinâmico para sistemas homogêneos de longo-alcance, é caracterizado por uma distribuição gaussiana de velocidades e esse é o aspecto que vamos considerar no presente trabalho. 


\subsection{Dinâmica Rumo ao Equilíbrio no Modelo de Hertel-Thirring - Generalizado}

O fato de que o termo colisional nas equações cinéticas de Boltzmann, Landau e Balescu-Lenard se anula para sistemas unidimensionais de muitas partículas ainda não foi muito bem explorado. Muitas tentativas de obter a descrição correta da cinética desses sistemas foram feitas [52] [18], mas nenhuma se mostrou completamente satisfatória por avaliarem parâmetros com muito ruído (como a magnetização) ou mesmo por serem feitas com números relativamente pequenos de partículas. Recentemente, Rocha Filho [28] e colaboradores de nosso grupo obtiveram uma equação de Landau generalizada válida para o acoplamento fraco que prevê de maneira correta o scaling da dinâmica desses sistemas como uma função do número de partículas. Isso foi comprovado por meio de simulações computacionais bastante robustas [28].

Em outubro de 2013, apresentamos um trabalho no XIII Latin American Workshop on Nonlinear Phenomena, sobre uma classe especial de potenciais de longo-alcance que nos permitiram investigar sua dinâmica utilizando simulações com número de partículas muito grandes (maiores que os utilizados anteriormente).

O potencial de interação aos pares foi escrito na forma

$$
V\left(x_{i}-x_{j}\right)=-u\left(x_{i}\right) u\left(x_{j}\right)
$$


em que $u(x)$ é uma função de $x$, generalizando o modelo proposto por Hertel e Thirring [34] para discutir o calor específico negativo na Astrofísica. Segue a Hamiltoniana do sistema

$$
H=-\sin \left(x_{i}\right) \sin \left(x_{j}\right) .
$$

As simulações computacionais foram realizadas utilizando um código paralelizado em GPU (CUDA) desenvolvido em nosso grupo. Confirmamos os resultados de Rocha Filho para um intervalo mais amplo de partículas.

A natureza simples deste modelo foi fundamental para discutirmos alguns problemas importantes ainda em aberto no campo das interações de longoalcance, tais como o surgimento de uma relaxação não-linear, além é claro, e principalmente, da dependência temporal entre o número de partículas e a dinâmica do sistema. Provamos por meio dessas simulações, para energias próximas à transição de fase, que o tempo de permanência no estado quasiestacionário de fato depende do número de partículas presentes no sistema. Quando a condição inicial é não-homogênea, o sistema evolui em um tempo proporcional a $1 / N$. Caso ela seja homogênea, o tempo de evolução depende de $1 / N^{2}$.

\subsubsection{Resultados Inspiradores}

Apresentamos os resultados encontrados nas simulações realizadas para o modelo de Hertel-Thirring generalizado [34]. A energia por partícula foi 
escolhida para ser próxima da transição de fase $e=0.71$, um Runge-Kutta de quarta ordem [50] foi utilizado na integração numérica com um passo de tempo de $\delta t=0.01$ e o erro máximo admitido foi de $10^{-4}$. Estudamos o quarto momento estatístico da velocidade nas simulações e a análise será apresentada para condições iniciais tanto não-homogênas como homogêneas.

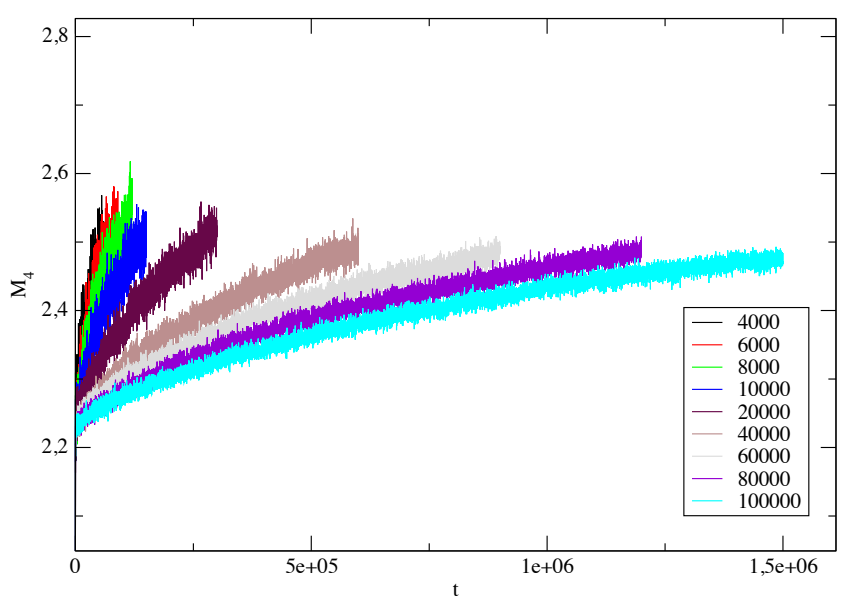

Figura 1.1: Quarto Momento $\left\langle M_{4}\right\rangle$ da velocidade como função do tempo para o modelo da Hamiltoniana da equação (1.25) com uma condição inicial nãohomogênea e energia por partícula 0.71 . A simulação foi feita para $N=4000$ até $N=100000$ partículas e está sem reescala do tempo.

Da Fig (1.1) a (1.5), podemos ver claramente que a reescala adequada para o tempo é proporcional a $1 / N$ quando a condição inicial é nãohomogênea e $1 / N^{2}$ para a condição homogênea. Atribuímos a segurança deste resultado à possibildade de efetuar simulações com números grandes 

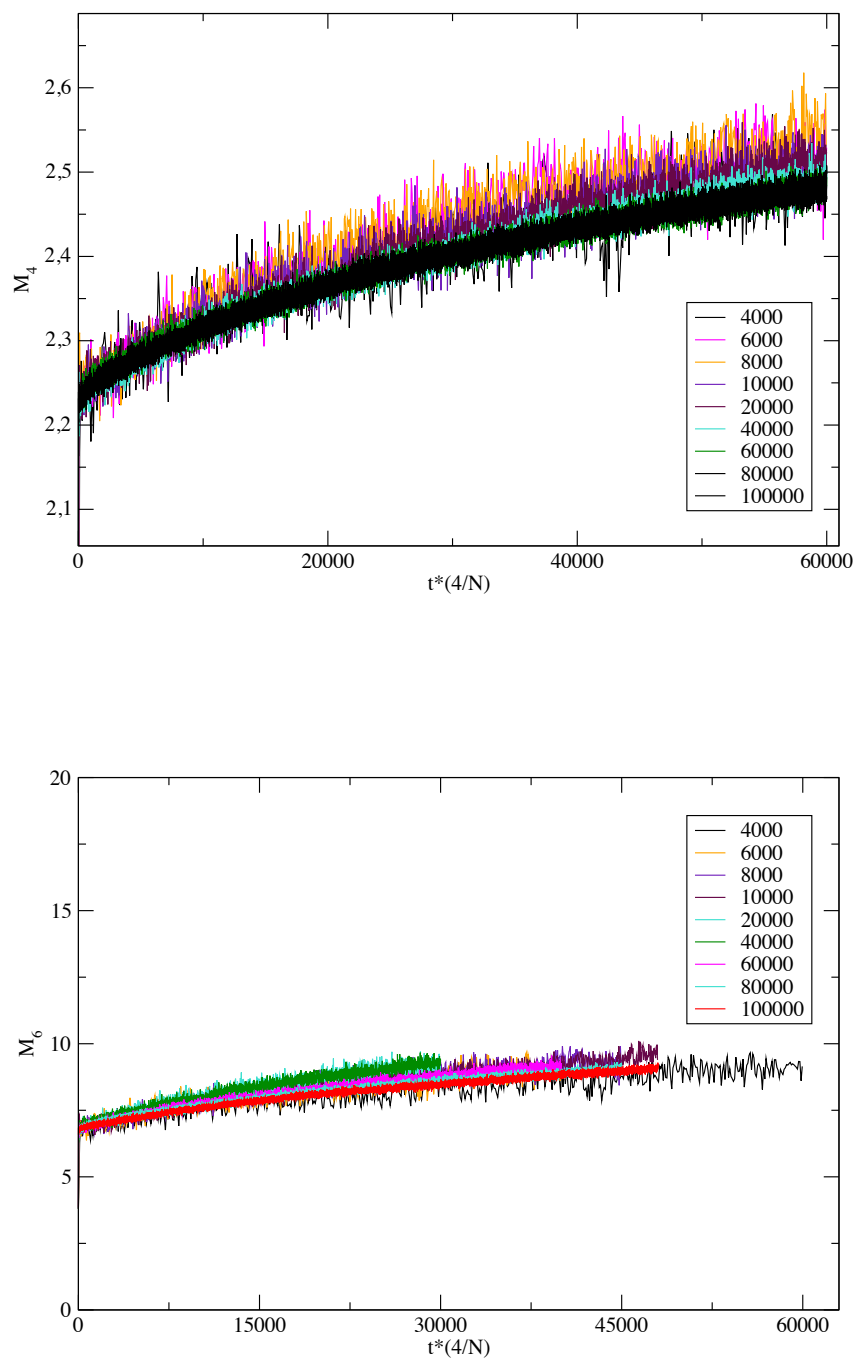

Figura 1.2: Quarto e sexto momentos $\left\langle M_{4}\right\rangle$ e $\left\langle M_{6}\right\rangle$ da velocidade como função do tempo para o modelo da Hamiltoniana da equação (1.25) com uma condição inicial não-homogênea e energia por partícula 0.71. A simulação foi feita para $N=4000$ até $N=100000$. No primeiro e no segundo quadros uma reescala com o fator $1 / N$ utilizada. 


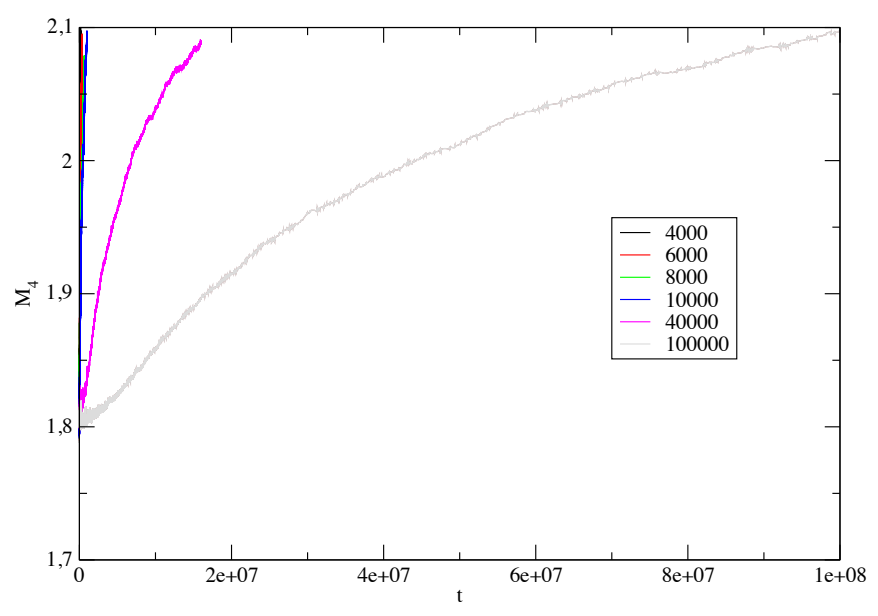

Figura 1.3: Quarto Momento $\left\langle M_{4}\right\rangle$ da velocidade como função do tempo para o modelo da hamiltonina da equação (1.25) com uma condição inicial homogênea e energia por partícula 0.71. A simulação foi feita para $N=4000$ até $N=100000$.

de partículas em tempo razoável, graças à simulação em GPU. Esse resultado reforça os obtidos ns referências [47, 28] para os modelos HMF e do anel.

Os resultados obtidos para o modelo de Hertel-Thirring generealizado serviram como norteadores para os estudos com os demais sistemas apresentados neste trabalho. A dependência entre o número de partículas e o tempo da dinâmica dos sistemas realmente acontece obedecendo o expoente 2. Isso aconteceria com outros sistemas físicos? Qual a origem desse comportamento? O estudo é apresentado nos próximos capítulos. 

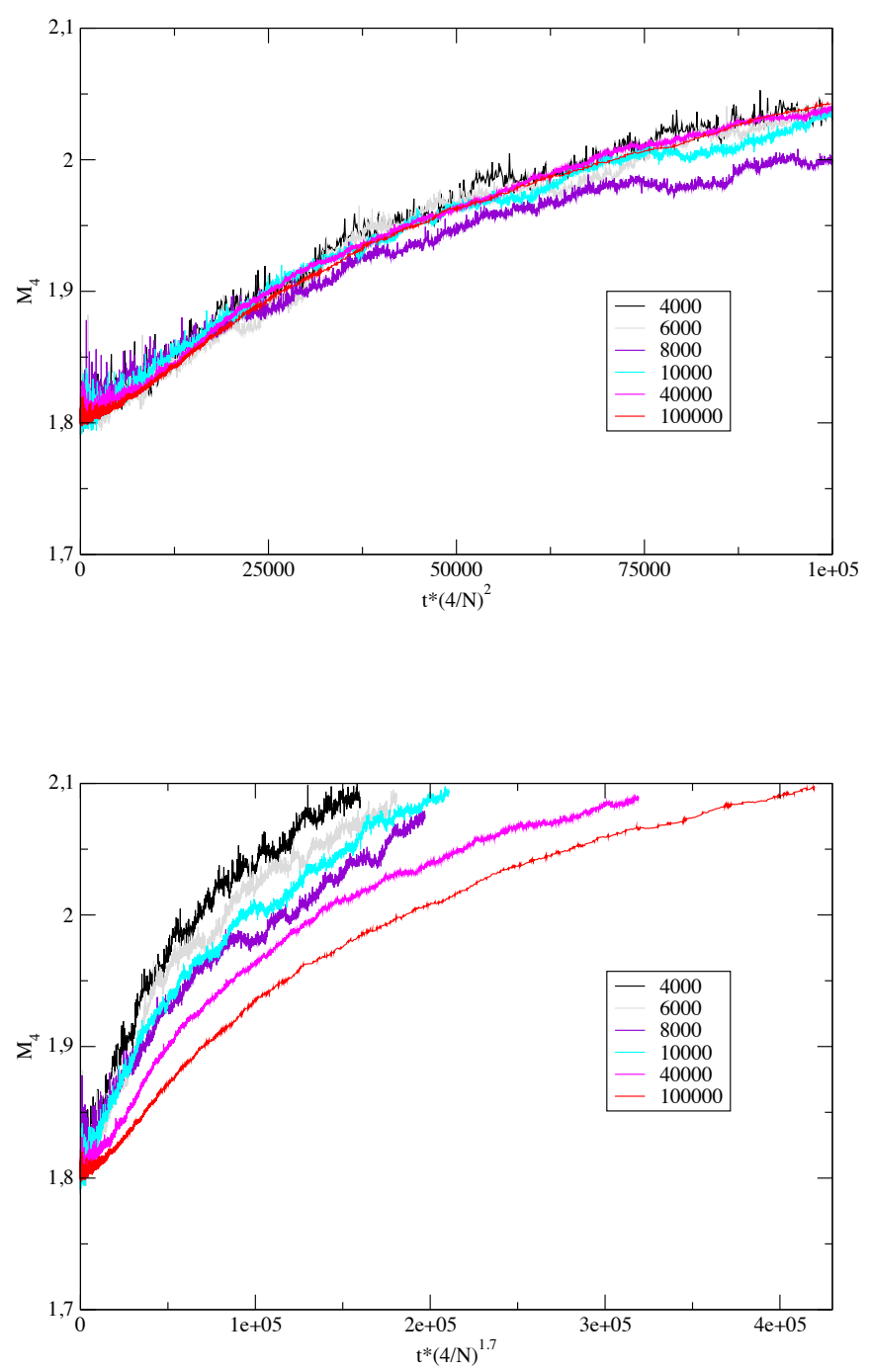

Figura 1.4: Quarto Momento $\left\langle M_{4}\right\rangle$ da velocidade como função do tempo para o modelo da Hamiltoniana da equação (1.25) com uma condição inicial homogênea e energia por partícula 0.71. A simulação foi feita para $N=$ 4000 até $N=100000$. No primeiro e no segundo quadros a reescala foi feita proporcional a $1 / N^{2}$ e $1 / N^{1.7}$, respectivamente. 

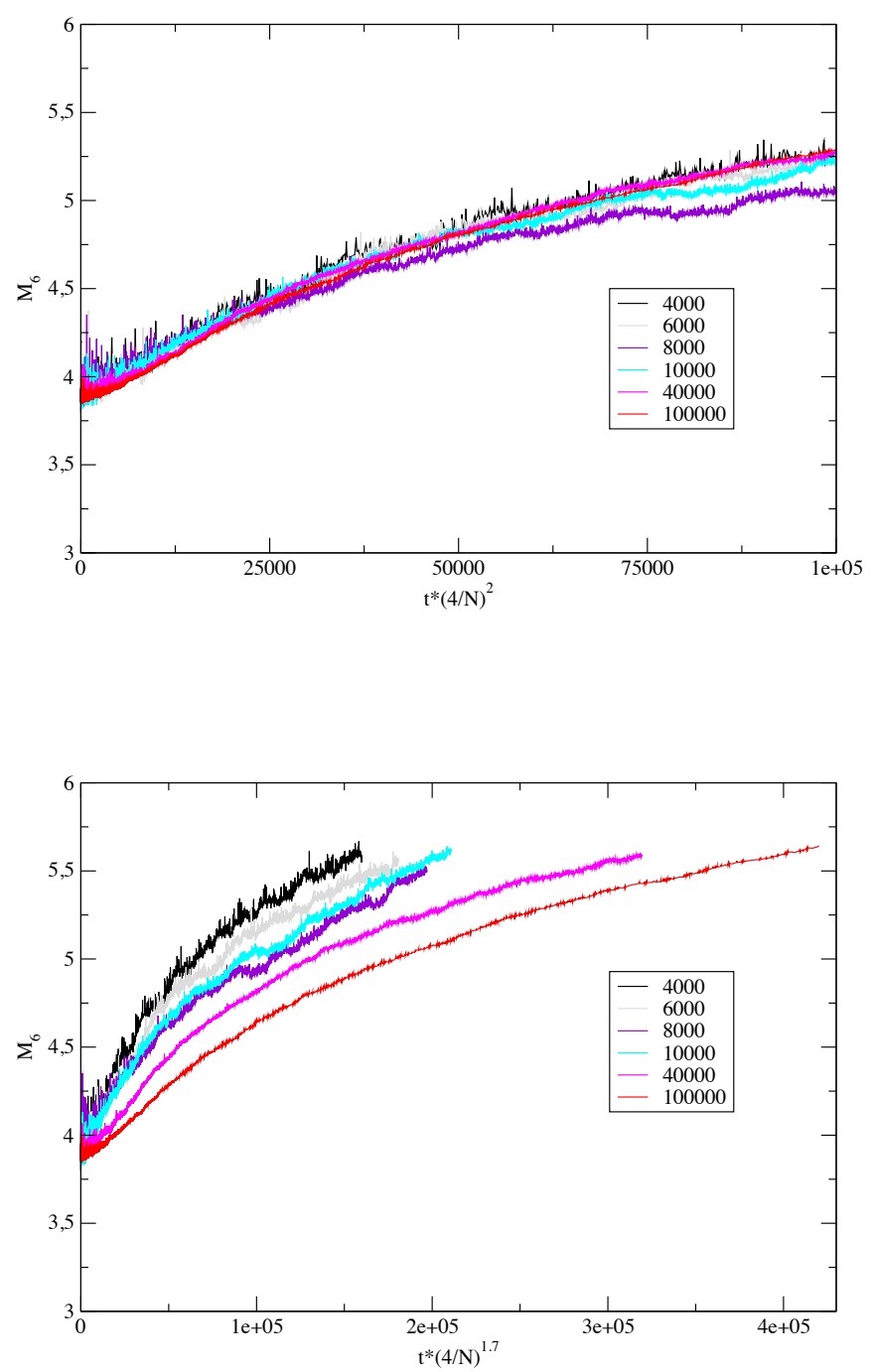

Figura 1.5: Sexto Momento $\left\langle M_{6}\right\rangle$ da velocidade como função do tempo para o modelo da Hamiltoniana da equação (1.25) com uma condição inicial homogênea e energia por partícula 0.71. A simulação foi feita para $N=4000$ até $N=100000$. No primeiro e no segundo quadros a reescala foi feita proporcional a $1 / N^{2}$ e $1 / N^{1.7}$, respectivamente. 


\section{Capítulo 2}

\section{O Modelo Anisotrópico de Campo}

\section{Médio}

\subsection{Apresentação do Modelo}

O modelo de estudo consiste de N spins clássicos de Heisenberg $\vec{S}_{i}$, globalmente acoplados, de comprimento unitário. Em termos de coordenadas polares com ângulos $\theta_{i}$ e $\phi_{i}$ pertencentes aos intervalos $[0, \pi]$ e $[0,2 \pi]$ respectivamente, as componentes do spin escrevem-se na forma $S_{i x}=\sin \left(\theta_{i}\right) \cdot \cos \left(\phi_{i}\right)$, $S_{i y}=\sin \left(\theta_{i}\right) \cdot \sin \left(\phi_{i}\right)$ e $S_{i z}=\cos \left(\theta_{i}\right)[30]$.

Nossa Hamiltoniana é:

$$
H=-\frac{J}{2 N} \sum_{i, j=1}^{N} \vec{S}_{i} \cdot \vec{S}_{j}+D \sum_{i=1}^{N} S_{i z}^{2}
$$


vamos fazer $J=1, D>0$ e $k_{B}=1$ a fim de reproduzir o modelo estudado em [30]. O fator $1 / N$ que antecede o primeiro termo aparece para conferir extensividade ao sistema de acordo com a previsão de Kac. O sistema continua apresentando comportamento não-aditivo uma vez que não pode ser trivialmente dividido em partes macroscópicas independentes, como poderia um sistema com interações de curto-alcance. Vamos considerar

$$
m=\left(m_{x}{ }^{2}+m_{y}{ }^{2}+m_{z}{ }^{2}\right)^{1 / 2},
$$

e

$$
\vec{m}=\langle\vec{S}\rangle=\frac{1}{N} \sum_{i=1}^{N} \vec{S}_{i},
$$

$\operatorname{com} m_{x}=\sin (\theta) \cdot \cos (\phi), m_{y}=\sin (\theta) \cdot \sin (\phi)$ e $m_{z}=\cos (\theta)$.

\subsubsection{O Equilíbrio}

Sabemos que sistemas com interações de longo-alcance evoluem rumo ao verdadeiro equilíbrio termodinâmico passando por uma relaxação violenta (muito rápida) e por um estado quasi-estacionário, cuja duração depende do número de partículas do sistema. Neste capítulo, estamos interessados na etapa final atingida: o equilíbrio termodinâmico, no qual a distribuição de velocidades é Gaussiana. O equilíbrio deste modelo é estudado na Ref [30], no presente trabalho, analisaremos o equilíbrio utilizando outra metodologia.

A fim de estudar o equilíbrio do sistema pela maximização de sua entropia 
[23], faremos

$$
A=\frac{1}{N} \sum_{i=1}^{N} S_{i z}{ }^{2}
$$

para escrever a energia na forma

$$
E=-N \cdot \vec{m} \cdot \vec{m}+D \cdot N \cdot A=N \cdot[-\vec{m} \cdot \vec{m}+D \cdot A]
$$

de forma que $m_{x}, m_{y}, m_{z}$ e $A$ serão nossas variáveis globais. A energia por partícula toma então a forma

$$
\begin{aligned}
e=E / N= & -\int f\left(\theta^{\prime}, \phi^{\prime}\right) \cdot m_{x} \cdot m_{x}^{\prime} \cdot \sin \left(\theta^{\prime}\right) d \theta^{\prime} d \phi^{\prime} \\
& -\int f\left(\theta^{\prime}, \phi^{\prime}\right) \cdot m_{y} \cdot m_{y}^{\prime} \cdot \sin \left(\theta^{\prime}\right) d \theta^{\prime} d \phi^{\prime} \\
& -\int f\left(\theta^{\prime}, \phi^{\prime}\right) \cdot m_{z} \cdot m_{z}^{\prime} \cdot \sin \left(\theta^{\prime}\right) d \theta^{\prime} d \phi^{\prime} \\
& +\int f\left(\theta^{\prime}, \phi^{\prime}\right) \cdot D \cdot A \cdot \sin \left(\theta^{\prime}\right) d \theta^{\prime} d \phi^{\prime}
\end{aligned}
$$

assumindo

$$
\int f(\theta, \phi) \sin (\theta) d \theta d \phi=1,
$$

em que consideramos a isotropia do espaço e a dependência angular das variáveis do sistema.

A entropia por partícula deve ser escrita como

$$
s=S / N=-\int f(\theta, \phi) \ln f(\theta, \phi) \sin (\theta) d \theta d \phi,
$$


que maximizada nos dará

$$
-\ln f(\theta, \phi)-1-\lambda-\beta . e(\theta, \phi)=0,
$$

com $e(\theta, \phi)$ a energia por partícula antes calculada e $\lambda$ e $\beta$ representando os multiplicadores de Lagrange. $\lambda$ e $\beta$ surgem quando se utiliza o Método dos Multiplicadores de Lagrange para se encontrar extremos (máximos e mínimos) de uma função, como é o caso. Substituindo a parte da energia por partícula, (2.7) assume a forma

$$
\begin{aligned}
& -\ln f(\theta, \phi)-1-\lambda+\beta\left[\quad m_{x} \quad \cdot \int f\left(\theta^{\prime}, \phi^{\prime}\right) \cdot m_{x}^{\prime} \cdot \sin \left(\theta^{\prime}\right) d \theta^{\prime} d \phi^{\prime}\right. \\
& +m_{y} \cdot \int f\left(\theta^{\prime}, \phi^{\prime}\right) \cdot m_{y}^{\prime} \cdot \sin \left(\theta^{\prime}\right) d \theta^{\prime} d \phi^{\prime} \\
& +m_{z} \cdot \int f\left(\theta^{\prime}, \phi^{\prime}\right) \cdot m_{z}^{\prime} \cdot \sin \left(\theta^{\prime}\right) d \theta^{\prime} d \phi^{\prime} \\
& \text { - } \left.\quad D \quad \cdot \int f\left(\theta^{\prime}, \phi^{\prime}\right) \cdot A \cdot \sin \left(\theta^{\prime}\right) d \theta^{\prime} d \phi^{\prime}\right]=0 .
\end{aligned}
$$

Na equação acima, vamos fazer $-\ln f(\theta, \phi)$ igual a $W(\theta, \phi)$ e o termo $(-1-\lambda)$ igual a $K$. Assim, reescrevendo a equação teremos

$$
W(\theta, \phi)+K+\beta\left[m_{x} \cdot \sigma_{x}+m_{y} \cdot \sigma_{y}+m_{z} \cdot \sigma_{z}-D \cdot \sigma_{A}\right]=0 .
$$

Note que os $\sigma$ da equação acima representam as integrais da equação anterior e são exatamente as componentes médias de $\vec{m}$. Vamos agora determinar a expressão para $f(\theta, \phi)$. 


$$
f(\theta, \phi)=e^{K+\beta\left[m_{x} \cdot \sigma_{x}+m_{y} \cdot \sigma_{y}+m_{z} \cdot \sigma_{z}-D . \sigma_{A}\right]}
$$

e, consequentemente,

$$
f(\theta, \phi)=K^{\prime} e^{\beta\left[m_{x} \cdot \sigma_{x}+m_{y} \cdot \sigma_{y}+m_{z} \cdot \sigma_{z}-D . \sigma_{A}\right]},
$$

$\operatorname{com} K^{\prime}=e^{K}$.

Nosso problema agora restringe-se a calcular os $\sigma$ que nos darão exatamente a magnetização média $(\langle\vec{m}\rangle)$ no equilíbrio. Escolhemos o eixo $x$ na direção de magnetização final de forma que teremos de determinar apenas $\sigma_{x}$ e $\sigma_{A}$.

Começando pelo $\sigma_{A}$, teremos:

$$
\sigma_{A}=\int f\left(\theta^{\prime}, \phi^{\prime}\right) D A \sin \left(\theta^{\prime}\right) d \theta^{\prime} d \phi^{\prime}=\int f\left(\theta^{\prime}, \phi^{\prime}\right) D\left(\sum_{i=1}^{N} \cos ^{2}\left(\theta_{i}^{\prime}\right)\right) \sin \left(\theta^{\prime}\right) d \theta^{\prime} d \phi^{\prime}
$$

Já utilizando a orientação do eixo $x$ como a dominante no equilíbrio e conhecendo a expressão para $f\left(\theta^{\prime}, \phi^{\prime}\right)$, a equação acima se transforma em

$$
\sigma_{A}=\int K^{\prime} e^{\beta\left[m_{x} \sigma_{x}-D \sigma_{A}\right]} D A \sin \left(\theta^{\prime}\right) d \theta^{\prime} d \phi^{\prime}
$$

que ainda precisa ser normalizada e assume, finalmente, a forma

$$
\sigma_{A}=\frac{\int K^{\prime} e^{\beta\left[m_{x} \sigma_{x}-D \sigma_{A}\right]} D\left(\sum_{i=1}^{N} \cos ^{2}\left(\theta_{i}\right)\right) \sin \left(\theta^{\prime}\right) d \theta^{\prime} d \phi^{\prime}}{\int K^{\prime} e^{\beta\left[m_{x} \sigma_{x}-D \sigma_{A}\right]} \sin \left(\theta^{\prime}\right) d \theta^{\prime} d \phi^{\prime}}
$$


Analisando a equação acima para os valores médios no equilíbrio, vemos que o $\left\langle\sigma_{A}\right\rangle$ é exatamente o valor médio de $\cos ^{2}\left(\theta_{i}\right)$, sobre todas as partículas e sobre todo o espaço angular (chamaremos $\left.\left\langle\cos ^{2} \theta\right\rangle\right)$.

Partiremos para a análise de $\sigma_{x}$. Sabendo que $\left\langle m_{x}\right\rangle=\langle\sin (\theta) \cdot \cos (\phi)\rangle$, podemos escrever o $\left\langle\sigma_{x}\right\rangle$ normalizado na forma

$$
\left\langle\sigma_{x}\right\rangle=\frac{\int e^{\beta\left[\sin \left(\theta^{\prime}\right) \cos \left(\phi^{\prime}\right)\left\langle\sigma_{x}\right\rangle-D \cos ^{2}\left(\theta^{\prime}\right)\right]} \sin ^{2}\left(\theta^{\prime}\right) \cos \left(\phi^{\prime}\right) d \theta^{\prime} d \phi^{\prime}}{\int e^{\beta\left[\sin \left(\theta^{\prime}\right) \cos \left(\phi^{\prime}\right)\left\langle\sigma_{x}\right\rangle-D \cos ^{2}\left(\theta^{\prime}\right)\right]} \sin \left(\theta^{\prime}\right) d \theta^{\prime} d \phi^{\prime}} .
$$

A equação acima não possui solução analítica, de forma que devemos expandi-la em torno de $\left\langle m_{x}\right\rangle$, na região próxima à temperatura crítica, até primeira ordem, para obter

$\left\langle\sigma_{x}\right\rangle \cdot\left(\int e^{\beta_{c} D \cos ^{2}\left(\theta^{\prime}\right)} \sin \left(\theta^{\prime}\right) d \theta^{\prime} d \phi^{\prime}-\beta_{c} \int e^{\beta D \cos ^{2}\left(\theta^{\prime}\right)} \sin ^{3}\left(\theta^{\prime}\right) \cos ^{2}\left(\phi^{\prime}\right) d \theta^{\prime} d \phi^{\prime}\right)=0$.

A temperatura crítica pode ser obtida igualando-se o termo entre parênteses a zero e deve satisfazer a relação a seguir

$$
\frac{2}{\beta_{c}}=1-\frac{1}{2 \beta_{c} D}+\frac{e^{-\beta_{c} D}}{\sqrt{\pi \beta_{c} D} \operatorname{Erf}\left[\sqrt{\beta_{c} D}\right]},
$$

em que $\operatorname{Erf}[x]=2 / \sqrt{\pi} \int_{0}^{x} e^{-t^{2}} d t$.

A densidade de energia crítica, por sua vez, $e_{c}=\left\langle\cos ^{2}(\theta)\right\rangle$, é da forma

$$
e_{c}=D\left(1-\frac{2}{\beta_{c}}\right)
$$


que é exatamente a densidade de energia para a qual acontece a transição de fase.

\subsubsection{Relaxação Rumo ao Equilíbrio}

A evolução temporal deste modelo é governada pelo conjunto de equações diferenciais de primeira ordem a seguir:

$$
\begin{aligned}
& \dot{S}_{i x}=S_{i y} \cdot m_{z}-S_{i z} \cdot m_{y}-2 \cdot D \cdot S_{i x} \cdot S_{i z} \\
& \dot{S}_{i y}=S_{i z} \cdot m_{x}-S_{i x} \cdot m_{z}+2 \cdot D \cdot S_{i x} \cdot S_{i z} \\
& \dot{S}_{i z}=S_{i x} \cdot m_{y}-S_{i y} \cdot m_{x}
\end{aligned}
$$

que estão em concordância com as equações da Mecânica Quântica obtidas quando se toma o limite para infinitos spins.

Para obter as equações acima, utilizamos o fato de que as derivadas temporais correspondem a

$$
\frac{d \vec{S}_{i}}{d t}=\left\{\vec{S}_{i}, H\right\}
$$

$\operatorname{com} i=1,2, \ldots N$.

Da equação para $\dot{S}_{i z}$, podemos concluir que $m_{z}$ é uma constante do movimento. Utilizando todas as equações já aprsentadas nesta sessão e relembrando que o parêntese de Poisson tem a forma

$$
\{A, B\}=\sum_{i=1}^{N}\left(\frac{\partial A}{\partial \phi_{i}} \frac{\partial B}{\partial S_{i z}}-\frac{\partial A}{\partial S_{i z}} \frac{\partial B}{\partial \phi_{i}}\right)
$$


para este modelo, podemos perceber, das equações de Hamilton, que

$$
\dot{\phi}=\frac{\partial H}{\partial S_{i z}}
$$

e

$$
\dot{S}_{i z}=-\frac{\partial H}{\partial \phi}
$$

As coordenadas generalizadas para este modelo são, por fim, $\phi_{i}$ e $S_{i z}$. Em termos das coordenadas generalizadas, a Hamiltoniana do sistema assume a forma

$$
\begin{aligned}
H= & -\left(\sqrt{1-S_{i z}^{2}}\right) \cos \left(\phi_{i}\right) \cdot \frac{1}{N} \sum_{j=1}^{N}\left(\sqrt{1-S_{j z}^{2}}\right) \cos \left(\phi_{j}\right) \\
& -\left(\sqrt{1-S_{i z}^{2}}\right) \sin \left(\phi_{i}\right) \cdot \frac{1}{N} \sum_{j=1}^{N}\left(\sqrt{1-S_{j z}^{2}}\right) \sin \left(\phi_{j}\right) \\
& -S_{i z} \cdot \frac{1}{N} \sum_{j=1}^{N} S_{j z}+D \cdot S_{i z}^{2},
\end{aligned}
$$

ou, de maneira um pouco mais simplificada, reescrita em termos das coordenadas generalizadas de posição $\left(\phi_{i}\right)$ e momento $\left(S_{i z}\right)$

$$
H=-\left(\sqrt{1-S_{i z}^{2}}\right) \cos \left(\phi_{i}\right) \cdot m_{x}-\left(\sqrt{1-S_{i z}^{2}}\right) \sin \left(\phi_{i}\right) \cdot m_{y}-S_{i z} \cdot m_{z}+D \cdot S_{i z}^{2},
$$

já que as componentes de $\vec{m}$ dependem dos ângulos $\theta$ e $\phi$ para cada partícula e quando é feita a média sobre todas elas, cada uma das componentes se torna uma constante do sistema. 


\subsection{O Estado Quasi-estacionário}

Realizamos várias simulações numéricas a fim de avaliar a dependência temporal existente entre o número de partículas $(N)$ presentes em nosso sistema e o tempo de duração do estado quasi-estacionário (o scaling da dinâmica do sistema), entre ouras características. A simulação foi elaborada levando-se em consideração a energia do sistema em termos das coordenadas generalizadas e sua evolução temporal foi governada pelas equações

$$
\dot{\theta}=m_{x} \sin \left(\phi_{i}\right)-m_{y} \cos \left(\phi_{i}\right)
$$

e

$$
\dot{\phi}=m_{x} \cot \left(\theta_{i}\right) \cos \left(\phi_{i}\right)+m_{y} \cot \left(\theta_{i}\right) \sin \left(\phi_{i}\right)-m_{z}+2 D \cos \left(\theta_{i}\right) .
$$

O parâmtero D foi escolhido para ser $D=15$ (o mesmo de [30]) e utilizamos um Runge-Kutta de quarta ordem na integração numérica [50]. A linguagem de programação utilizada (CUDA) permite a simulação com números muito grandes de partículas e passos muito curtos em tempos razoáveis, de forma que o erro acumulado nos resultados obtidos é muito pequeno. Em uma realização como a apresentada com 1000000 de partículas, leva-se aproximadamente 100 horas. Veja a Fig. (2.1).

Este resultado representa a evolução dinâmica do quarto momento estatístico da variável $\theta$ ao longo do tempo. Pode-se inferir que há interação 


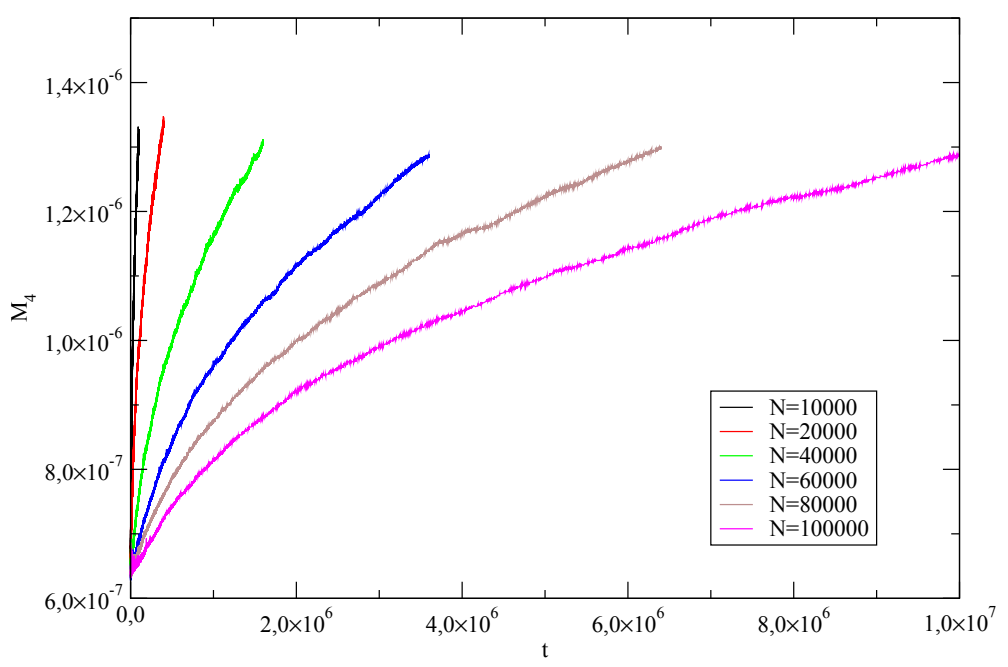

Figura 2.1: Quarto momento estatístico $\left\langle M_{4}\right\rangle$ da variável $\theta$ para $N=10000$ até $N=100000$ de um sistema homogêneo com energia $e=0.24$ sem reescala do tempo.

dinâmica no sistema pelo comportamento evolutivo dessa variável.

\subsubsection{Os Efeitos de N Finito}

A fim de estudar a dinâmica de um estado homogêneo para efeitos de comparação, utilizamos as mesmas condições inciais da referência [30]: um "waterbag"(distribuição uniforme) nos intervalos $\phi \in[0,2 \pi)$ e $\theta \in[\pi / 2-$ $a, \pi / 2+a]$, com energia por partícula

$$
e=\frac{D}{3} \sin ^{2} a
$$

e escolhemos $a$ tal que $e=0.24$ (mais uma vez replicando os parâmetros de [30]). O estado é espacialmente homogêneo e energeticamente estável para 
esta escolha de energia. Da Ref. [28] a reescala esperada para a dinâmica desse QSS é $N^{2}$. Gupta e Mukamel, por outro lado, obtiveram uma reescala $N^{1.7}$ de suas simulações numéricas. Argumentamos que, de maneira semelhante ao que ocorre no modelo HMF, o expoente 1.7 só acontece porque o número de partículas estudado na simulação foi muito pequeno. Utilizando-se um numero de partículas suficientemente grande, a reescala se torna proporcional a $N^{2}$.

Em um estado homogêneo, a distribuição espacial para a variável $\phi$ é sempre uniforme (a despeito de pequenas flutuações), mas a distribuição para a variável $\theta$ varia lentamente ao longo do tempo rumo ao equilíbrio termodinâmico [28]. Como consequência disso, a dinâmica pode ser provada pela evolução temporal dos momentos estatísticos $M_{n}=\left\langle(\theta-\langle\theta\rangle)^{n}\right\rangle$. Nota-se que há uma evolução ao longo do tempo evidenciando a dinâmica para os momentos pares. Momentos ímpares de $\theta$ se anulam para uma distribuição par em $\theta$, como acontece neste caso. A Figura (2.2) mostra o segundo momento $M_{2}$ como função do tempo. Ele varia muito lentamente para o estado considerado (é praticamente uma constante do movimento), portanto consideramos a evolução temporal do quarto momento estatístico, que responde melhor a pequenas mudanças no estado estatístico do sistema. Na Ref. [30] Gupta e Mukamel consideraram a média $\langle\cos \theta\rangle$ em que é mais difícil de caracaterizar as pequenas modificações na função de distribuição de $\theta$ (compare, por exemplo, Fig. 3 do artigo deles a nossas figuras (2.2) e (2.1).

As equações de movimento nas Eq. (2.27 e 2.28) são solucionadas uti- 


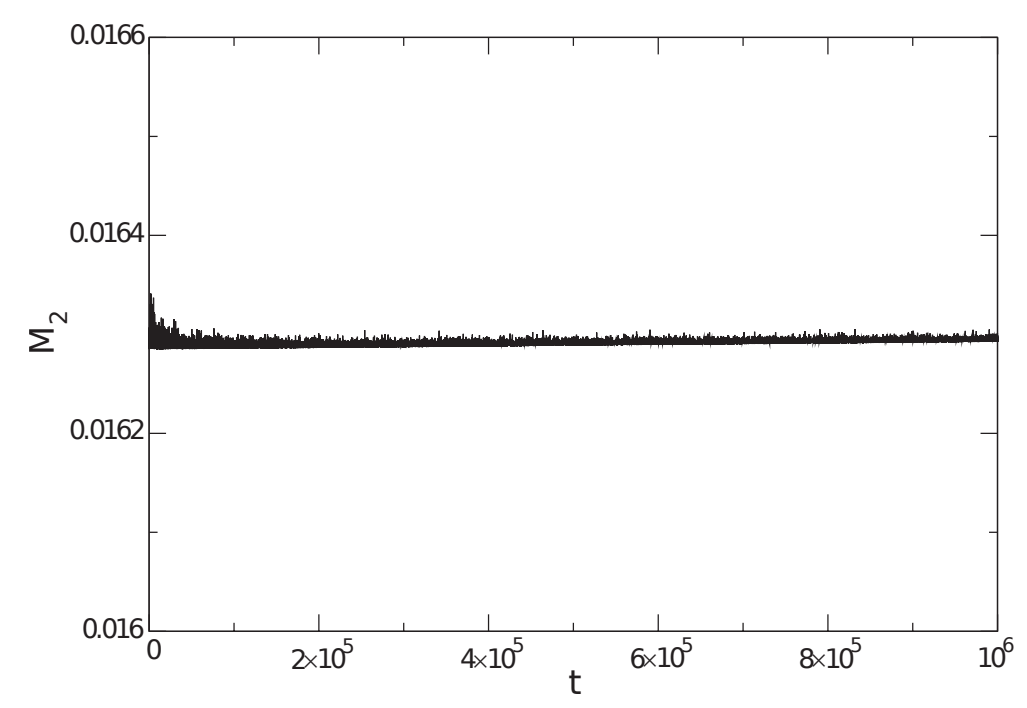

Figura 2.2: Momento $\left\langle M_{2}\right\rangle$ da variável $\theta_{i}$ como função do tempo para $N=$ 100000 do sistema homogêneo com energia $e=0,24$.

lizando uma implementação paralela de um algoritmo de Runge-Kutta de quarta ordem [50] em uma unidade de processamento gráfico utilizando a extensão CUDA da linguagem C [22]. Isso nos permite fazer simulações com um número muito maior de partículas do que os considerados em Ref. [30] (na referência citada o maior número de partículas foi 5000 ao passo que em nosso estudo chegamos a realizar simulações com 1000000 de partículas). O passo de tempo utilizado é $\delta t=0.01$ e garante um erro relativo máximo na energia de $10^{-4}$. A figura (2.1) mostra a evolução temporal de $M_{4}$ para diferentes números de partículas até $N=100000$. As figuras (2.3) e (2.4) mostram o mesmo resultado, mas com as reescalas $1 / N^{1.7}$ e $1 / N^{2}$ no tempo, respectivamente. O melhor colapso dos dados é, claramente, o obtido com a reescala $N^{2}$. Note como as curvas se sobrepõem melhor com a reescala $N^{2}$. 


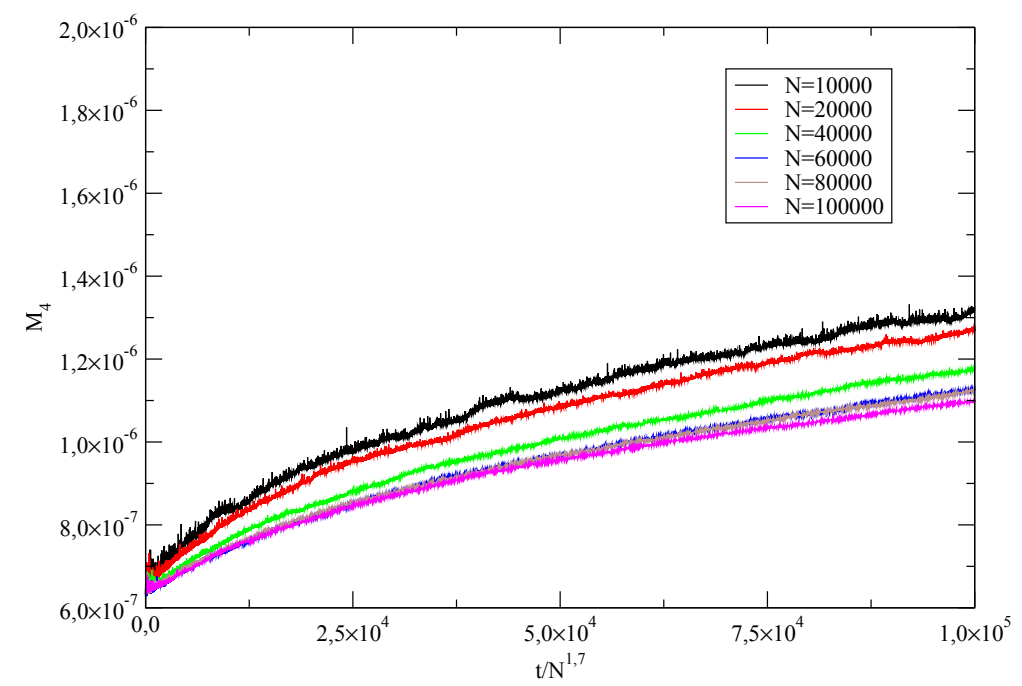

Figura 2.3: Momento $\left\langle M_{4}\right\rangle$ da variável $\theta_{i}$ como função do tempo para diferentes números de partículas $N=10000$ até $N=100000$ com reescala na forma $N^{-1,7}$ do sistema homogêneo com energia $e=0,24$.

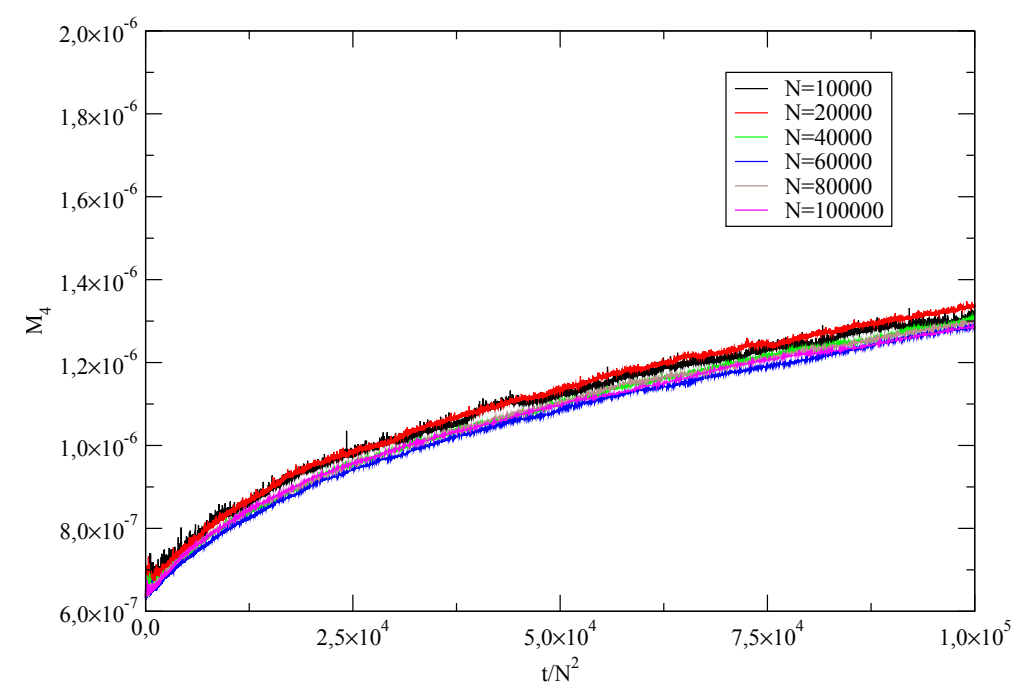

Figura 2.4: $\mathrm{O}$ mesmo que na Fig. (2.3) mas com a reescala do tempo na forma $N^{-2}$ do sistema homogêneo com energia $e=0,24$. 
Para comparar quantitativamente nossos resultados apresentados com os resultados anteriores, realizamos uma série de simulações para o mesmo número de partículas como na Ref. [30] mas considerando também valores de $N$ até 60000 . Fazendo a média sobre muitas realizações, comparamos a evolução temporal $M_{4}$ para um determinado valor de $N$ com os $N$ anteriores nas simulações e fizemos um ajuste de mínimos quadrados para a diferença entre as séries temporais reescaladas com os dois tipos de $1 / N^{\delta}$.

Os resultados são apresentados na Tabela 2.1 e corroboram, a menos de alguns pequenos desvios, que a reescala ideal é $N^{2}$. Para construir a tabela, utilizamos o Método dos Quadrados Mínimos, ou Quadrados Mínimos Ordinários (MQO) (ou OLS - do inglês, Ordinary Least Squares). Este método é uma técnica de otimização matemática que procura encontrar o melhor ajuste para um conjunto de dados tentando minimizar a soma dos quadrados das diferenças entre o valor estimado e os dados observados. Consiste em um estimador (que foi programado em $C$ ) que minimiza a soma dos quadrados dos resíduos da regressão, de forma a maximizar o grau de ajuste do modelo aos dados observados. Veja o apêndice B.

A figura (2.5) mostra o momento estatístico $M_{4}$ para os mesmos números de partículas apresentados na Tabela (2.1) com o tempo reescalado com $1 / N^{2}$ e um colapso muito bom para $N \geq 5000$.

Notamos que, na Ref. [30], Gupta e Mukamel determinaram o comportamento da reescala considerando os valores $N=300,1000,3000,5000$. A diferença dos resultados obtidos por eles e dos nossos resultados para os ca- 


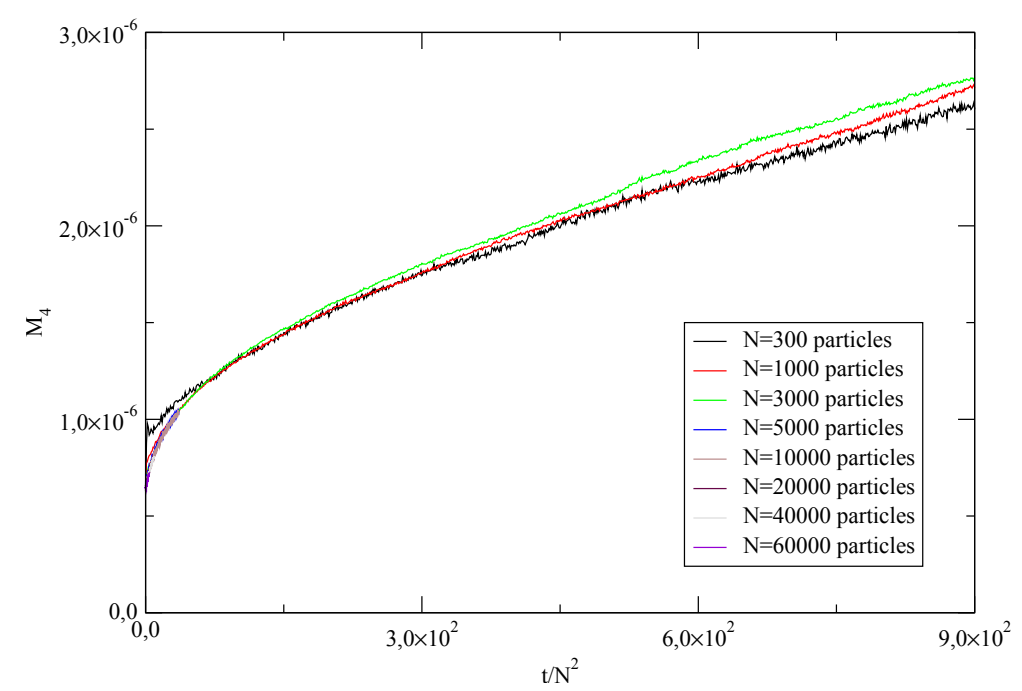

Figura 2.5: O mesmo que na Fig. (2.3) mas com a reescala do tempo na forma $N^{-2}$.

sos $N=3000$ e 5000 derivam do fato de que considerar a magnetização como uma variável relevante leva a resultados mais imprecisos que quando se considera os momentos estatísticos das variáveis de momento (veja também a discussão na Ref. [22]).

\begin{tabular}{|c|c|c|}
$N_{1}$ & $N_{2}$ & $\delta$ \\
\hline \hline 300 & 1000 & 1.767 \\
1000 & 3000 & 1.797 \\
3000 & 5000 & 2.015 \\
5000 & 10000 & 2.056 \\
10000 & 20000 & 2.072 \\
20000 & 40000 & 2.066 \\
40000 & 60000 & 2.096
\end{tabular}

Tabela 2.1: Melhor reescala em $N^{\delta}$ para o momento $M_{4}$ entre um par de dados simulados com $N_{1}$ e $N_{2}$ partículas.

Os resultados apresentados até agora, somados aos do próximo capítulo 
foram publicados em [41]. 


\section{Capítulo 3}

\section{Teoria Cinética}

Neste capítulo nos restringiremos a tratar da Teoria Cinética para sistemas com interações de longo-alcance.

As equações de evolução da a função reduzida a uma partícula que podem ser derivadas da equação de Liouville a partir da hierarquia BBGKY e da correlação dinâmica, se obedecem às condições que provocam o aparecimento de um termo colisional na equação fechada para essa evolução, formam o grupo de equações características da Teoria Cinética.

A equação cinética mais simples utilizada na Física de Partículas é a equação de Vlasov comentada em uma das seções da nossa Introdução. Vlasov não deduziu sua equação, introduziu-a como um postulado.

Rostoker e Rosenbluth [49] mostraram que a equação de Vlasov pode ser deduzida da equação de Liouville, via hierarquia de equações BBGKY, como uma aproximação, quando desprezadas as funções de correlação. Nessa 
aproximação, as interações entre as partículas são levadas em consideração apenas por meio do campo médio auto-consistente, tipo Hartree. A equação de Vlasov é invariante por inversão temporal.

Em 1960, Balescu [5] e Lenard [38], trabalhando independentemente um do outro e utilizando métodos diferentes, deduziram uma equação cinética para plasmas, levando em consideração funções correlação a duas partículas e desprezando as de ordem mais elevada. A equação de Balescu-Lenard não é invariante por inversão temporal e, portanto, só pode descrever processos irreversíveis.

Para se obter a equação de Balescu-Lenard, a hierarquia BBGKY é truncada na segunda equação, por meio da hipótese de correlações fracas. A forma assintótica da função correlação a duas partículas é determinada com base nas hipóteses de Bogoliubov [11] sobre a hierarquia de tempos de relaxação.

Além das equações de Vlasov e Balescu-Lenard, trataremos neste trabalho das equações de Boltzmann e Landau.

\subsection{A Hierarquia BBGKY}

Antes de passarmos a derivação das Equações Cinéticas, vamos comentar o ponto de partida para as equações de Boltzmann, Landau e Balescu-Lenard: a Hierarquia BBGKY.

Considere um sistema clássico formado por $N$ partículas interagentes con- 
finadas em um volume $V$. Se o sistema possui uma Hamiltoniana na forma

$$
H=\sum_{j=1}^{N} H^{0}\left(x_{j}\right)+\sum_{j<k=1}^{N} V\left(x_{j}, x_{k}\right)
$$

em que $H^{0}$ é a parte livre e $V_{j k} \equiv V\left(x_{j}, x_{k}\right)$, o termo de interação, então a evolução dinâmica do sistema é dada pela equação de Liouville em sua forma exata

$$
\partial_{t} F=\sum_{j=1}^{N} \mathscr{L}_{j}^{0} F+\sum_{j<n}^{N} \mathscr{L}_{j n}^{\prime} F,
$$

com $F=F\left(x_{1} \ldots x_{N}\right)$ representando a distribuição do espaço de fase e $\mathscr{L}_{j}$, o operador Liouvilliano. A conservação de partículas requer a seguinte condição de normalização

$$
\int d x_{1} \ldots d x_{N} F\left(x_{1} \ldots x_{N} ; t\right)=1
$$

Tomando-se a derivada ao longo do tempo

$$
\partial_{t} \int d x_{1} \ldots d x_{N} F\left(x_{1} \ldots x_{N} ; t\right)=0
$$

e substituindo a Eq. (3.2) em Eq. (3.4), tem-se

$$
\int d x_{1} \ldots d x_{N}\left(\sum_{j=1}^{N} \mathscr{L}_{j}^{0}+\sum_{j<n}^{N} \mathscr{L}_{j n}^{\prime}\right) F=0
$$

que deve ser válida independente do número de partículas. Dessa forma, cada 
termo da Eq. (3.5) deve se anular

$$
\begin{aligned}
& \int d x_{1} \ldots d x_{N} \sum_{j=1}^{N} \mathscr{L}_{j}^{0} F=0 \\
& \int d x_{1} \ldots d x_{N} \sum_{j<n}^{N} \mathscr{L}_{j n}^{\prime} F=0 .
\end{aligned}
$$

Buscando deduzir uma equação de evolução apropriada para a função distribuição reduzida a $s$ partículas

$$
f_{s}\left(x_{1} \ldots x_{s}\right)=\frac{N !}{(N-s) !} \int d x_{s+1} \ldots d x_{N} F
$$

e, utilizando as equações anteriores, escrevemos

$$
\begin{aligned}
\partial_{t} f_{s}\left(x_{1} \ldots x_{s}\right) & =\frac{N !}{(N-s) !} \int d x_{s+1} \ldots d x_{N} F \\
& =\frac{N !}{(N-s) !} \int d x_{s+1} \ldots d x_{N}\left(\sum_{j=1}^{N} \mathscr{L}_{j}^{0}+\sum_{j<n}^{N} \mathscr{L}_{j n}^{\prime}\right) F
\end{aligned}
$$

Note que é possível separar o somatório contendo a parte livre do Liouvilliano $\mathcal{L}^{0}$ da seguinte maneira:

$$
\sum_{j=1}^{N} \mathscr{L}_{j}^{0}=\sum_{j=1}^{s} \mathscr{L}_{j}^{0}+\sum_{j=s+1}^{N} \mathscr{L}_{j}^{0} .
$$


Da equação (3.10) pode-se concluir que, se $j \in(1, \ldots, s)$, o operador $\mathscr{L}_{j}^{0}$ não será afetado pela integração, já que partimos de $d x_{s+1}$. Por outro lado, se $j \in(s+1, \ldots, N)$, segue da (3.6) que tais integrais serão nulas. Assim,

$$
\sum_{j=1}^{N} \frac{N !}{(N-s) !} \int d x_{s+1} \ldots d x_{N} F=\sum_{j=1}^{s} \mathscr{L}_{j}^{0} f_{s}\left(x_{1}, \ldots, x_{s}\right)
$$

Na parte que contém $\mathscr{L}_{j n}^{\prime}$ da Eq. (3.9), a análise seria a semelhante, mas com as seguintes possibilidades:

- $j, n \in(1, \ldots, s)$ : aqui o $\mathscr{L}_{j n}^{\prime}$ pode ser colocado fora da integral e obtemos

$$
\sum_{j<n=1}^{s} \mathscr{L}_{j n}^{\prime} f_{s}\left(x_{1}, \ldots, x_{s}\right)
$$

- $j, n \in(s+1, \ldots, N)$ : segue pela Eq. (3.6) que tais integrais serão nulas;

- $j \in(1, \ldots, s)$ e $n(>j) \in(s+1, \ldots, N)$ : neste caso, integrando sobre $x_{n}$ e devido à simetria da função $F$, escreve-se

$$
\begin{aligned}
& \frac{N !}{(N-s) !} \int d x_{s+1} \ldots d x_{N} \sum_{j=1}^{s} \sum_{n=s+1}^{N} \mathscr{L}_{j n}^{\prime} F\left(x_{1} \ldots x_{N}\right) \\
= & \sum_{j=1}^{s} \int d x_{s+1} \mathscr{L}_{j, s+1}^{\prime} f_{s+1}\left(x_{1} \ldots x_{s+1}\right) .
\end{aligned}
$$


Por fim, a hierarquia pode ser escrita como $[6,56]$

$$
\begin{aligned}
\partial_{t} f_{0} & =0 \\
\partial_{t} f_{1}\left(x_{1}\right) & =\mathscr{L}_{1}^{0} f_{1}\left(x_{1}\right)+\int d x_{2} \mathscr{L}_{12}^{\prime} f_{2}\left(x_{1}, x_{2}\right) \\
\partial_{t} f_{s}\left(x_{1} \ldots x_{s}\right) & =\left(\sum_{j=1}^{s} \mathscr{L}_{j}^{0}+\sum_{j<} \sum_{n=1}^{s} \mathscr{L}_{j n}^{\prime}\right) f_{s}\left(x_{1} \ldots x_{s}\right) \\
& +\sum_{j=1}^{s} \int d x_{s+1} \mathscr{L}_{j, s+1}^{\prime} f_{s+1}\left(x_{1} \ldots x_{s+1}\right) \text { com } 2 \leq s ;
\end{aligned}
$$

As equações anteriories evidenciam a estrutura da hierarquia: a função distribuição reduzida a $s$ partículas depende da função distribuição de ordem $s+1$.

Diferentes formas de truncar a hieraquia conduzem às equações cinéticas

1 . Equação de Boltzmann;

2 . Equação de Landau;

3 . Equação de Balescu-Lenard.

Para derivarmos as equações acima, basta que sejam feitas considerações sobre as escalas temporais e de comprimento em face das colisões entre partículas para cada tipo de sistema. 


\subsection{Equação de Boltzmann}

A equação de Boltzmann é uma equação de movimento para a função distribuição a uma partícula adequada para um gás ideal. A seguir, derivaremos a equação seguindo a linha da obra de Boltzmann.

No limite em que não existem interações, ou seja, as partículas são independentes entre si, $F(\mathbf{x}, \mathbf{v}, t)$ (normalizada para o número total de partículas, $N)$ satisfaz a equação de Liouville a uma partícula. Esta equação implica que o número de partículas que entram no elemento $\delta v \delta x$ do espaço de fase seguindo a trajetória de uma partícula, no intervalo de tempo $\delta t$, é zero. Denotando esse número por $\delta R$, podemos escrever

$$
\delta R=\delta \mathbf{x} \delta \mathbf{v} \delta t\left(\frac{\partial F}{\partial t}+\mathbf{v} \cdot \frac{\partial F}{\partial \mathbf{x}}+\frac{\mathbf{K}}{m} \cdot \frac{\partial F}{\partial \mathbf{v}}\right)=0
$$

em que $\mathbf{K}$ é um campo externo. Considere agora que as partículas interagem. Especificamente, faremos $r_{0}$ representando o alcance da interação. Esse parâmetro é definido de modo que, para uma distância $r$ entre partículas tal que $r>r_{0}$ a interação desaparece. Quando as partículas entram no "domínio de interação" $\left(r<r_{0}\right)$, elas colidem. Façamos a distância média entre as colisões igual a $l$ (essa distância será chamada de livre caminho médio). Nosso primeiro critério para a validade da derivação da equação de Boltzmann que sergue será

$$
l>>r_{0} .
$$


Esse vínculo garante que as trajetórias sejam retilíneas entre as colisões. Seja $V$ a velocidade do fluxo térmico (ou velocidade típica das partículas como em [6]), pode-se introduzir os tempos

$$
\tau=\frac{l}{V}
$$

$\mathrm{e}$

$$
\tau_{0}=\frac{r_{0}}{V}
$$

de tal forma que nossa primeira condição de validade pode ser reescrita como $\tau>>\tau_{0}$.

Se passarmos a considerar as interações, então a equação de Liouville a uma partícula sofre alterações em virtude das colisões entre partículas. No presente modelo, esse fenômeno é representado pela taxa líquida com que as colisões modificam o número de partículas que entram no (ou saem do) elemento de volume $\delta v \delta x$ do espaço de fase. Podemos escrever, portanto

$$
\delta R=\delta R_{+}-\delta R_{-}
$$

em que $\delta R_{+}$reprensenta o número de partículas que entram no elemento de volume $\delta v \delta x$ num intervalo de tempo $\delta t$ e $\delta R_{-}$, o número de partículas que saem do mesmo elemento.

Primeiro vamos considerar $\delta R_{-}$. A velocidade de todas as partículas pode ser dividida em dois grupos: uma pequena faixa de velocidades que 
se enquadram no intervalo $\delta v$ e todas as outras velocidades denotadas pela variável $v_{1}$. O número de partículas que são ejetadas do elemento $\delta v \delta x$ do espaço de fase no tempo $\delta t$ é simplesmente o número total de colisões que as partículas de $v$ têm com todas as outras partículas (isto é, as partículas $\left.v_{1}\right)$ no tempo $\delta t$. Segue-se que, para calcular $\delta R_{-}$devemos levar em conta todas as colisões entre pares de partículas que lançam uma delas para fora do elemento de volume considerado. Esse número de partículas pode ser escrito matematicamente como

$$
\delta R_{-}=\int_{l} F_{2}\left(\mathbf{z}, \mathbf{z}_{1}\right) \delta \mathbf{v}_{1} \delta \mathbf{v} \delta \mathbf{x}
$$

em que $\mathbf{z}=(\mathbf{x}, \mathbf{v})$ e $F_{2}$ foi normalizada em $N(N-1)$ com $N$ sendo o número total de partículas (para melhor entender o que representa cada um dos termos da expressão acima, visite a Ref [39]).

O elemento $\delta R_{+}$terá uma forma semelhante, porém será gerado por colisões inversas

$$
\delta R_{+}=\int_{l^{\prime}} F_{2}\left(\mathbf{z}^{\prime}, \mathbf{z}_{1}^{\prime}\right) \delta \mathbf{v}_{1}^{\prime} \delta \mathbf{v}^{\prime} \delta \mathbf{x}^{\prime} .
$$

O próximo passo para nossa derivação é retomar as integrais invariantes de Poincaré. Podemos escrever

$$
\delta \mathbf{v} \delta \mathbf{x} \delta \mathbf{v}_{1} \delta \mathbf{x}_{1}=\delta \mathbf{v}^{\prime} \delta \mathbf{x}^{\prime} \delta \mathbf{v}_{1}^{\prime} \delta \mathbf{x}_{1}^{\prime} .
$$


Substituindo esse resultado nas equações (3.17) e (3.18), pode-se chegar a

$$
\frac{\partial F}{\partial t}+\mathbf{v} \cdot \frac{\partial F}{\partial \mathbf{x}}+\frac{\mathbf{K}}{m} \cdot \frac{\partial F}{\partial \mathbf{v}}=\int\left[F_{2}\left(\mathbf{z}^{\prime}, \mathbf{z}_{1}^{\prime}\right)-F_{2}\left(\mathbf{z}, \mathbf{z}_{1}\right)\right] d \mathbf{v}_{1} \sigma d \Omega
$$

em que o termo $d \Omega$ está associado ao elemento de ângulo sólido na demonstração em [39] e $\sigma$ é a sessão de choque. Assumindo que $F_{2}$ é homogênea nas dimensões do domínio de colisões, temos

$$
\begin{gathered}
F_{2}\left(\mathbf{z}, \mathbf{z}_{1}\right)=F_{2}\left(\mathbf{v}, \mathbf{v}_{1}\right) \\
F_{2}\left(\mathbf{z}^{\prime}, \mathbf{z}_{1}^{\prime}\right)=F_{2}\left(\mathbf{v}^{\prime}, \mathbf{v}_{1}^{\prime}\right) .
\end{gathered}
$$

Impondo a condição de caos molecular em que as partículas de um gás ideal não são correlacionadas, podemos escrever

$$
\begin{gathered}
F_{2}\left(\mathbf{v}, \mathbf{v}_{1}\right)=\frac{N-1}{N} F(\mathbf{v}) F\left(\mathbf{v}_{1}\right) \\
F_{2}\left(\mathbf{v}^{\prime}, \mathbf{v}_{1}^{\prime}\right)=\frac{N-1}{N} F\left(\mathbf{v}^{\prime}\right) F\left(\mathbf{v}_{1}^{\prime}\right)
\end{gathered}
$$

e a equação de Boltzmann, finalmente, é a equação que pode ser escrita na forma

$$
\frac{\partial F}{\partial t}+\mathbf{v} \cdot \frac{\partial F}{\partial \mathbf{x}}+\frac{\mathbf{K}}{m} \cdot \frac{\partial F}{\partial \mathbf{v}}=\int\left[F\left(\mathbf{v}^{\prime}\right) F\left(\mathbf{v}_{1}^{\prime}\right)-F(\mathbf{v}) F\left(\mathbf{v}_{1}\right)\right] d \mathbf{v}_{1} d \Omega
$$

em que o termo colisional é o termo à direita da equação. Em sistemas 
unidimensionais e homogêneos $v=v^{\prime}$ e $v_{1}=v_{1}^{\prime}$ e, portanto, o termo colisional se anula.

\subsection{Equação de Landau}

A equação de Landau, segundo [39] pode ser derivada por meio de uma transformação a partir da equação de Fokker-Planck a seguir

$$
\frac{\partial f}{\partial t}+\mathbf{v} \cdot \frac{\partial f}{\partial \mathbf{x}}+\frac{\partial}{\partial \mathbf{v}} \alpha f-\frac{1}{2} \frac{\overline{\overline{\partial^{2}}}}{\partial \mathbf{v} \partial \mathbf{v}}: \overline{\bar{b}} f=0
$$

em que $\alpha$ é um coeficiente de friç̧ão correpondente à dissipação de Rayleigh

e $\overline{\bar{b}}$ é um tensor de difusão.

Para demonstrar a transformação necessária, antes vamos estabelecer algumas propriedades tensoriais da velocidade relativa

$$
\mathbf{g}=\mathbf{v}_{1}-\mathbf{v}
$$

e o tensor

$$
\overline{\bar{T}}=g^{2} \overline{\bar{I}}-\overline{\overline{g g}} .
$$

Podemos escrever as seguintes relações

$$
\frac{\partial}{\partial v_{\mu}} g_{\nu}=-\delta_{\mu \nu}
$$




$$
\begin{gathered}
\nabla \cdot \mathbf{g}=\frac{\partial}{\partial v_{\mu}} g_{\mu}=-3 \\
\frac{\partial}{\partial v_{\mu}} g=\frac{\partial}{\partial v_{\mu}}\left(g^{2}\right)^{1 / 2}=\frac{1}{2}\left(g^{2}\right)^{-1 / 2} \frac{\partial}{\partial v_{\mu}} g_{\nu} g_{\nu}=\frac{2 g_{\nu}}{2 g} \frac{\partial g_{\nu}}{\partial v_{\mu}}=-\frac{g_{\mu}}{g} \\
\frac{\partial}{\partial v_{\mu}} g^{2}=-2 g_{\nu} \frac{\partial g_{\nu}}{\partial v_{\mu}}=-2 g_{\mu} .
\end{gathered}
$$

Seja $f(g)$ uma função escalar, então

$$
\frac{\partial f(g)}{\partial v_{\mu}}=-\frac{\partial f(g)}{\partial v_{1 \mu}}
$$

e podemos partir para a determinação de $\nabla \cdot \overline{\bar{T}}$

$$
\begin{aligned}
\frac{\partial}{\partial v_{\mu}} T_{\nu} & =\frac{\partial}{\partial v_{\mu}} \delta_{\mu \nu} g^{2}-\frac{\partial}{\partial v_{\mu}} g_{\mu} g_{\nu} \\
& =\frac{\partial}{\partial v_{\mu}} g^{2}-g_{\mu} \frac{\partial}{\partial v_{\mu}} g_{\nu}-g_{\nu} \frac{\partial}{\partial v_{\mu}} g_{\mu} \\
& =-2 g_{\nu}+g_{\nu}+3 g_{\nu}=2 g_{\nu}
\end{aligned}
$$

Combinando os resultados, escrevem-se

$$
\begin{aligned}
\nabla \cdot \overline{\bar{T}} & =2 \mathbf{g} \\
\mathbf{g} \cdot \overline{\bar{T}} & =0 \\
\nabla \cdot f(g) \overline{\bar{T}} & =f(g) \nabla \cdot \overline{\bar{T}} .
\end{aligned}
$$


Vamos agora reescrever a equação de Fokker-Planck como

$$
\begin{aligned}
\frac{1}{K} \frac{D f}{D t} & =-2 \frac{\partial}{\partial v_{\nu}} f \int \frac{g_{\nu}}{g^{3}} f_{1} d \mathbf{v} \frac{1}{2} \frac{\partial}{\partial v_{\nu}} \frac{\partial}{\partial v_{\mu}} f \int \frac{T_{\mu \nu} f_{1} d \mathbf{v}_{1}}{g^{3}} \\
& =\frac{\partial}{\partial v_{\nu}}\left[-2 f \int \frac{g_{\nu}}{g^{3}} f_{1} d \mathbf{v}+\frac{1}{2} \frac{\partial}{\partial v_{\mu}} f \int \frac{T_{\mu \nu} f_{1} d \mathbf{v}_{1}}{g^{3}}\right]
\end{aligned}
$$

e vamos expandir o segundo termo dentro dos colchetes para ficar com

$$
\frac{1}{2} \int d \mathbf{v}_{1} f_{1}\left(\frac{T_{\mu \nu}}{g^{3}} \frac{\partial}{\partial v_{\mu}} f+f \frac{\partial}{\partial v_{\mu}} \frac{T_{\mu \nu}}{g^{3}}\right)=\frac{1}{2} \int d \mathbf{v}_{1} f_{1}\left(\frac{T_{\mu \nu}}{g^{3}} \frac{\partial}{\partial v_{\mu}} f+2 \frac{f g_{\nu}}{g^{3}}\right)
$$

O segundo termo de (3.31) se combina com o primeiro termo dentro dos colchetes em (3.30) para nos dar

$$
\frac{1}{K} \frac{D f}{D t}=\frac{1}{2} \frac{\partial}{\partial v_{\mu}}\left(\int d \mathbf{v}_{1} \frac{T_{\mu \nu}}{g^{3}} f_{1} \frac{\partial}{\partial v_{\mu}} f-f \int f_{1} \frac{\partial}{\partial v_{\mu}} \frac{T_{\mu \nu}}{g^{3}} d \mathbf{v}_{1}\right)
$$

O segundo termo pode ser transformado da seguinte mandeira

$$
\begin{aligned}
-\int f_{1} \frac{\partial}{\partial v_{\mu}} \frac{T_{\mu \nu}}{g^{3}} d \mathbf{v}_{1} & =+\int f_{1} \frac{\partial}{\partial v_{1 \mu}}\left(\frac{T_{\mu \nu}}{g^{3}}\right) d \mathbf{v}_{1} \\
& =\int \frac{\partial}{\partial v_{1 \mu}}\left(\frac{l_{1} T_{\mu \nu}}{g^{3}}\right) d \mathbf{v}_{1}-\int \frac{T_{\mu \nu}}{g^{3}} \frac{\partial f_{1}}{\partial v_{1 \mu}} d \mathbf{v}_{1}
\end{aligned}
$$

e, ignorando o termo de superfície, teremos a equação de Landau na forma

$$
\frac{2}{K} \frac{D f}{D t}=\frac{\partial}{\partial v_{\mu}} \int d \mathbf{v}_{1} \frac{T_{\mu \nu}}{g^{3}}\left[f_{1} \frac{\partial f}{\partial v_{\mu}}-f \frac{\partial f_{1}}{\partial v_{1 \mu}}\right]
$$

Acabamos de apresentar a derivação tensorial da equação de Landau a partir 
de [39]. Em [6], no entanto, a derivação (será aprsentada no próximo capítulo) é tal que nos leva a forma

$$
\frac{\partial f\left(\mathbf{v}_{\mathbf{1}}, t\right)}{\partial t}=\frac{8 \pi^{4} n}{m^{2}} \int d \mathbf{v}_{2} \int d \mathbf{k} \tilde{V}^{2}(k)\left(\mathbf{k} \cdot \partial_{12}\right) \delta(\mathbf{k} \cdot \mathbf{g})\left(\mathbf{k} \cdot \partial_{12}\right) f\left(\mathbf{v}_{1}, t\right) f\left(\mathbf{v}_{2}, t\right) .
$$

Para chegar a esse formato, parte-se da Hierarquia BBGKY e adota-se a hipótese de Markovianização para garantir que o potencial seja diferente de zero somente na região específica em que a distância entre as partículas é menor que o comprimento de correlação $\left(r_{0}\right.$ - distância ao longo da qual duas partículas conseguem interagir significativamente). Em outras palavras, somente as faixas de valores que se econtram entre 0 e o comprimento de correlação $\left(r_{0}\right)$ e entre 0 e o tempo de correlação $\left(\tau_{0}\right)$ devem contribuir para a equação.

A primeira vista, a equação de Landau para um sistema homogêneo é bastante diferente da equação de Boltzmann [6]. Ao invés de uma equação de perda e ganho, encontramos um operador derivada-parcial o que nos remete a algo mais parecido com uma difusão. Na derivação original da equação de Landau, no entanto, em 1936, pode-se atribuir o comportamento de perda e ganho a uma segunda derivada da função distribuição reduzida com relação à velocidade.

A equação de Landau descreve um processo irreversível, assim como a equação de Balescu-Lenard que apresentamos a seguir. 


\subsection{Equação de Balescu-Lenard}

A equação de Lenard-Balescu é obtida da hierarquia BBGKY após algumas suposições: correlações entre três partículas podem ser desprezadas, o ensemble é espacialmente homogêneo, a correlação a duas partículas relaxa muito mais rápido que a função distribuição a uma partícula [48] (Hipótese de Markovianização). Ela tem a forma

$$
\begin{aligned}
\frac{\partial f(\mathbf{v}, t)}{\partial t}= & -\frac{8 \pi^{4} n_{0}}{m_{e}{ }^{2}} \nabla_{v} \cdot \int d \mathbf{k} d \mathbf{v}^{\prime} \mathbf{k} \mathbf{k} \cdot \frac{\phi^{2}(k)}{|\epsilon(\mathbf{k}, \mathbf{k} \cdot \mathbf{v})|^{2}} \\
& \cdot \delta\left[\mathbf{k} \cdot\left(\mathbf{v}-\mathbf{v}^{\prime}\right)\right]\left[f(\mathbf{v}) \nabla_{v^{\prime}} f\left(\mathbf{v}^{\prime}\right)-f\left(\mathbf{v}^{\prime}\right) \nabla_{\mathbf{v}} \mathbf{f}(\mathbf{v})\right]
\end{aligned}
$$

O lado direito dessa equação representa fisicamente o termo colisional (colisões a duas partículas), característica que se evidencia quando o termo $\phi(k) /|\epsilon(\mathbf{k}, \mathbf{k . v})|$ aparece elevado ao quadrado e representa o potencial de interação entre duas partículas.

De todas as equações apresentadas, note que a equação de Balescu-Lenard é a equação em que fica mais evidente o fato de que, no caso unidimensional, o termo colisional se anula. A delta de Dirac implica em $v^{\prime}=v$ e acaba por nos obrigar a ir à ordem seguinte na aproximação de campo fraco como foi feito em [28]. 


\section{Capítulo 4}

\section{Dinâmica de Estados Homogêneos}

Neste capítulo, nos restringiremos ao estudo de estados homogêneos unidimensionais.

Em muitos trabalhos anteriores, acreditava-se que o tempo da dinâmica de sistemas homogêneos com interações de longo-alcance escalava com $N^{-1,7}$ (ver [40] e referencias nela). Esse resultado é completamente discordante com as previsões matemáticas e teóricas, mas apesar disso, as imulações computacionais apontavam diretamente para ele. Ocorre que, quando o número de partículas é pequeno (não tende para infinito) o termo colisional de ordem $1 / N$ não se anula e acaba por influenciar o tempo da dinâmica. A dependência proporcional a 1,7 é, então, uma soma dos efeitos do termo de ordem $1 / N$ e do termo $1 / N^{2}$, como veremos a seguir.

Nossos resultados estão de acordo com o que se espera de uma teoria cinética derivada da hierarquia BBGKY em uma expansão em série de po- 
tências $1 / N$. Funções de correlação a duas e três partículas contribuem com termos proporcionais a $1 / N$ e $1 / N^{2}$, respectivamente. Como contribuições de duas partículas para a equação cinética se anulam neste caso, devemos considerar as contribuições de colisões a três partículas que são proporcionais a $1 / N^{2}$. Estas considerações são baseadas na introdução do factor de Kac na Hamiltoniana e a reescala proporcional a $N^{-1,7}$ obtida por Gupta e Mukamel é recuperada para pequenos valores de $N$. Esta escala incomum dá-se pela falha da hipótese markovianização para pequenos $N$ utilizada na determinação das equações Balescu-Lenard e Landau, que exige que a função de auto-correlação de força (para sistemas homogêneos) seja significativamente diferentes de zero apenas para tempos muito curtos, se comparados com a escala de tempo dinâmica sobre a qual a função-distribuição a uma partícula varia significativamente. Vamos mostrar isso explicitamente para o caso mais simples da equação Landau, i.e. para acoplamento fraco, da mesma maneira que essas aproximações são utilizadas na dedução da equação Balescu-Lenard (ver Ref. [6] para uma discussão mais aprofundada sobre estas suposições).

A função-distribuição a $N$ partículas $f_{n}\left(\mathbf{r}_{1}, \mathbf{v}_{1}, \ldots, \mathbf{r}_{N}, \mathbf{v}_{N} ; t\right)$ é a densidade de probabilidade em um espaço de fase a $N$-partículas para uma partícula no tempo $t$ ter posição $\mathbf{r}_{i}$ e momento $\mathbf{p}_{i}$.

Definindo a função distribuição a s-partículas como $f_{s} \equiv f_{s}\left(\mathbf{r}_{1}, \mathbf{v}_{1}, \ldots, \mathbf{r}_{s}, \mathbf{v}_{s} ; t\right)=\int d \mathbf{r}_{s+1} d \mathbf{v}_{s+1} \cdots d \mathbf{r}_{N} d \mathbf{v}_{N} f_{N}\left(\mathbf{r}_{1}, \mathbf{v}_{1}, \ldots, \mathbf{r}_{N}, \mathbf{v}_{N} ; t\right)$ 
em que $\mathbf{r}_{i}$ e $\mathbf{p}_{i}$ são os vetores posição e momento da partícula $i$ em $d$ dimensões. A equação de Liouville implica que a função distribuição reduzida satisfaz a hierarquia BBGKY [39, 6]:

$$
\frac{\partial}{\partial t} f_{s}=\sum_{j=1}^{s} \hat{K}_{j} f_{s}+\frac{1}{N} \sum_{j<k=1}^{s} \hat{\Theta}_{j k} f_{s}+\frac{N-s}{N} \sum_{j=1}^{s} \int d \theta_{s+1} d v_{s+1} \hat{\Theta}_{j, s+1} f_{s+1}
$$

em que

$$
\hat{K}_{j}=-\mathbf{v}_{j} \cdot \nabla_{j}, \quad \hat{\Theta}_{j k}=-\nabla_{j} V\left(\mathbf{r}_{j}-\mathbf{r}_{k}\right) \partial_{j k}, \quad \partial_{j k} \equiv \frac{\partial}{\partial \mathbf{v}_{j}}-\frac{\partial}{\partial \mathbf{v}_{k}}
$$

e $\nabla_{j}$ é o operados gradiente para a posição da partícula $j$. A fim de obter uma equação cinética fechada para a função distribuição a uma partícula $f_{1}$ temos que determinar a dependância funcional de $f_{2}$ em $f_{1}$ (hipótese de Bogolyubov [39]). Isso pode ser feito escrevendo-se a as funções distribuição reduzidas na forma de uma expansão em aglomerados, que para um sistema homogêneo é dada por:

$$
\begin{aligned}
f_{2}\left(\mathbf{v}_{1}, \mathbf{v}_{2}, \mathbf{r}_{1}-\mathbf{r}_{2}\right)= & f_{1}\left(\mathbf{v}_{1}\right) f_{1}\left(\mathbf{v}_{2}\right)+C_{2}\left(\mathbf{v}_{1}, \mathbf{v}_{2}, \mathbf{r}_{1}-\mathbf{r}_{2}\right), \\
f_{3}\left(\mathbf{v}_{1}, \mathbf{v}_{2}, \mathbf{v}_{3}, \mathbf{r}_{1}-\mathbf{r}_{2}, \mathbf{r}_{2}-\mathbf{r}_{3}\right)= & f_{1}\left(\mathbf{v}_{1}\right) f_{1}\left(\mathbf{v}_{2}\right) f_{1}\left(\mathbf{v}_{3}\right)+\sum_{P(1,2,3)} f_{1}\left(\mathbf{v}_{1}\right) C_{2}\left(\mathbf{v}_{2}, \mathbf{v}_{3}, \mathbf{r}_{2}-\mathbf{r}_{3}\right) \\
& +C_{3}\left(\mathbf{v}_{1}, \mathbf{v}_{2}, \mathbf{v}_{3}, \mathbf{r}_{1}-\mathbf{r}_{2}, \mathbf{r}_{2}-\mathbf{r}_{3}\right),
\end{aligned}
$$

e assim sucessivamente (a dependência temporal está implícita). $P(1,2,3)$ representa as permutações das partículas 1, 2 e 3 e $C_{s}$ é a função correlação a 
$s$-partículas. Vamos considerar o parâmetro $\lambda 1$ que caracteriza a intensidade da interação, i. e. $V=\mathcal{O}(\lambda)$. A correlação a duas partículas requer que a interação entre duas partículas seja criada e, portanto, $C_{2}$ é de ordem $\lambda$. Uma correlação a três partículas requer a interação entre dois pares de partículas e, assim, $C_{3}$ é de ordem $\lambda^{2}$ e assim sucessivamente. Considerando o caso $s=1$ na Eq. (4.2) e utilizando a Eq. (4.4) temos:

$$
\frac{\partial}{\partial t} f_{1}\left(\mathbf{v}_{1} ; t\right)=\frac{N-1}{N} \int d \mathbf{v}_{2} d \mathbf{r}_{2} \hat{\Theta}_{12}\left[f_{1}\left(\mathbf{v}_{1} ; t\right) f_{1}\left(\mathbf{v}_{2} ; t\right)+C_{2}\left(\mathbf{v}_{1}, \mathbf{v}_{2}, \mathbf{r}_{1}-\mathbf{r}_{2} ; t\right)\right] .
$$

A função correlação a duas partículas é a solução para a equação obtida substituindo a Eq. (4.5) em Eq. (4.2) para $s=2$ e descartando termos de ordem maior contendo correlações a três partículas:

$$
\left(\frac{\partial}{\partial t}-\hat{K}_{1}-\hat{K}_{2}\right) C_{2}\left(\mathbf{v}_{1}, \mathbf{v}_{2}, \mathbf{r}_{1}-\mathbf{r}_{2} ; t\right)=\hat{\Theta}_{12} f_{1}\left(\mathbf{v}_{1} ; t\right) f_{1}\left(\mathbf{v}_{2} ; t\right) .
$$

A solução pode ser escrita como:

$$
\begin{aligned}
C_{2}\left(\mathbf{v}_{1}, \mathbf{v}_{2}, \mathbf{r}_{1}-\mathbf{r}_{2} ; t\right)=e^{\left(\hat{K}_{1}+\hat{K}_{2}\right) t} C_{2}\left(\mathbf{v}_{1}, \mathbf{v}_{2}, \mathbf{r}_{1}-\mathbf{r}_{2} ; 0\right) \\
\quad+\int_{0}^{t} d t e^{\left(\hat{K}_{1}+\hat{K}_{2}\right) \tau} \hat{\Theta}_{12} f_{1}\left(\mathbf{v}_{1} ; t-\tau\right) f_{1}\left(\mathbf{v}_{2} ; t-\tau\right) .
\end{aligned}
$$

O primeiro termo à direita da Eq. (4.7) é um termo de transiente dado a correlações existentes em $t=0$ que se anula rapidamente [6]. Substituindo Eq. (4.8) e verificando que a força de campo médio se anula em um estado 
homogêneo, obtemos (utilizando $N-s \rightarrow N$ para $N$ grande):

$$
\begin{aligned}
\frac{\partial}{\partial t} f_{1}\left(\mathbf{v}_{1} ; t\right) & =\int_{0}^{t} d t \int d \mathbf{v}_{2} d \mathbf{r}_{2} \hat{\Theta}_{12} e^{\left(\hat{K}_{1}+\hat{K}_{2}\right) \tau} \hat{\Theta}_{12} f_{1}\left(\mathbf{v}_{1} ; t-\tau\right) f_{1}\left(\mathbf{v}_{2} ; t-\tau\right) \\
& =\int_{0}^{t} d t \int d \mathbf{v}_{2} d \mathbf{r}_{2} \partial_{12} \nabla_{1} V\left(\mathbf{r}_{12}\right) e^{\left(\hat{K}_{1}+\hat{K}_{2}\right) \tau} \nabla_{1} V\left(\mathbf{r}_{12}\right) \partial_{12} f_{1}\left(\mathbf{v}_{1} ; t-\tau\right) f_{1}\left(\mathbf{v}_{2} ; t-\tau\right) \\
& =\int_{0}^{t} d t \int d \mathbf{v}_{2} d \mathbf{r}_{2} \partial_{12} \nabla_{1} V\left(\mathbf{r}_{12}\right) \nabla_{1} V\left(\mathbf{r}_{12}-\mathbf{v}_{12} \tau\right) \partial_{12} f_{1}\left(\mathbf{v}_{1} ; t-\tau\right) f_{1}\left(\mathbf{v}_{2} ; t-\tau\right)
\end{aligned}
$$

com $\mathbf{r}_{12} \equiv \mathbf{r}_{1}-\mathbf{r}_{2}$ and $\mathbf{v}_{12} \equiv \mathbf{v}_{1}-\mathbf{v}_{2}$. A auto-correlação de força de $\mathbf{F}(\mathbf{r}, t)$ na posição $\mathbf{r}$ é definida por

$$
\mathcal{C}(t) \equiv\langle F(t) F(0)\rangle=\int d \mathbf{r} \mathbf{F}(\mathbf{r}, 0) \mathbf{F}(\mathbf{r}, t)=\int d \mathbf{r} \nabla V\left(\mathbf{r}-\mathbf{v}_{12} t\right) \nabla V(\mathbf{r}) .
$$

Consequentemente teremos:

$$
\frac{\partial}{\partial t} f_{1}\left(\mathbf{v}_{1} ; t\right)=\int_{0}^{t} d t \int d \mathbf{v}_{2} \partial_{12}\langle F(\tau) F(0)\rangle \partial_{12} f_{1}\left(\mathbf{v}_{1} ; t-\tau\right) f_{1}\left(\mathbf{v}_{2} ; t-\tau\right)
$$

Esta é uma equação mestra não-markoviana já que depende de $f_{1}$ no intervalo de 0 a $t$. Para obter uma verdadeira (markoviana) equação cinética, o procedimento ususal é assumir que a escala de tempo dinâmica $t_{d}$ ao longo da qual a função distribuição a uma partícula $f_{1}$ varia significativamente é muito maior que a escala de tempo $t_{c}$ (tempo de correlação) de forma que a auto-correlação de força seja suficientemente pequena. Neste caso, podemos substituir $f_{1}(\mathbf{v} ; t-\tau)$ no integrando da Eq. (4.10) por $f_{1}\left(\mathbf{v}_{1} ; t\right)$, que corresponde a aproximação balística (movimento livre para um sistema homogê- 
neo) e estender a integração no tempo para infinito. Finalmente obtemos a equação de Landau, que é uma equação puramente markoviana:

$$
\frac{\partial}{\partial t} f_{1}\left(\mathbf{v}_{1} ; t\right)=\int_{0}^{\infty} d t \int d \mathbf{v}_{2} \partial_{12}\langle F(\tau) F(0)\rangle \partial_{12} f_{1}\left(\mathbf{v}_{1} ; t\right) f_{1}\left(\mathbf{v}_{2} ; t\right) .
$$

Como discutido anteriormente, para um sistema homogêneo unidimensional, essas correções se anulam e devemos tomar a próxima ordem $1 / N$ na expansão. Normalmente, podemos considerar parte do processo de markovianização levar em conta escalas de tempo tais que $t_{d} \gg t_{c}$. Se essa condição falha, ente outras consequencias, temos o fato de que o termo colisional não se anula perfeitamente nos sistemas homogêneos unidimensionais e devemos esperar que isso afete a escala da dinâmica.

De forma a sustentar este aspecto, computamos a auto-correlação de força das simulações numéricas por:

$$
\mathcal{C}(t)=\frac{1}{N} \sum_{i=1}^{N} F_{i}(t) F_{i}(0)
$$

em que $F_{i}(t)$ é a força na partícula $i$ num tempo $t$ devida a todas as demais partículas.

Introduzindo a transformada de Fourier no potencial

$$
\tilde{V}(k)=1 / 2 \pi \int d r V(r) e^{-i k r}
$$


a equação de Landau pode ser escrita na forma equivalente:

$$
\begin{aligned}
\frac{\partial}{\partial t} f_{1}\left(\mathbf{v}_{1} ; t\right)= & 4 \pi^{2} \int d \mathbf{v}_{2} \int d \mathbf{k} \\
& \times \partial_{12} \cdot \mathbf{k} \delta\left(\mathbf{k} \cdot \mathbf{v}_{12}\right)[\tilde{V}(k)]^{2} \mathbf{k} \cdot \partial_{12} f_{1}\left(\mathbf{v}_{1} ; t\right) f_{1}\left(\mathbf{v}_{2} ; t\right)
\end{aligned}
$$

Como já dissemos, no caso unidimensional, a delta de Dirac implica que $v_{2}=v_{1}$ e $\partial_{12}=0$. Assim, devemos tomar a ordem seguinte na aproximação de campo fraco ([28]). Como $V$ é também de ordem $1 / N$, isso implica que a dinâmica tem uma escala em $1 / N$ no caso não homogêneoe $1 / N^{2}$ no caso homogêneo (se o termo seguinte da expansão for não-nulo).

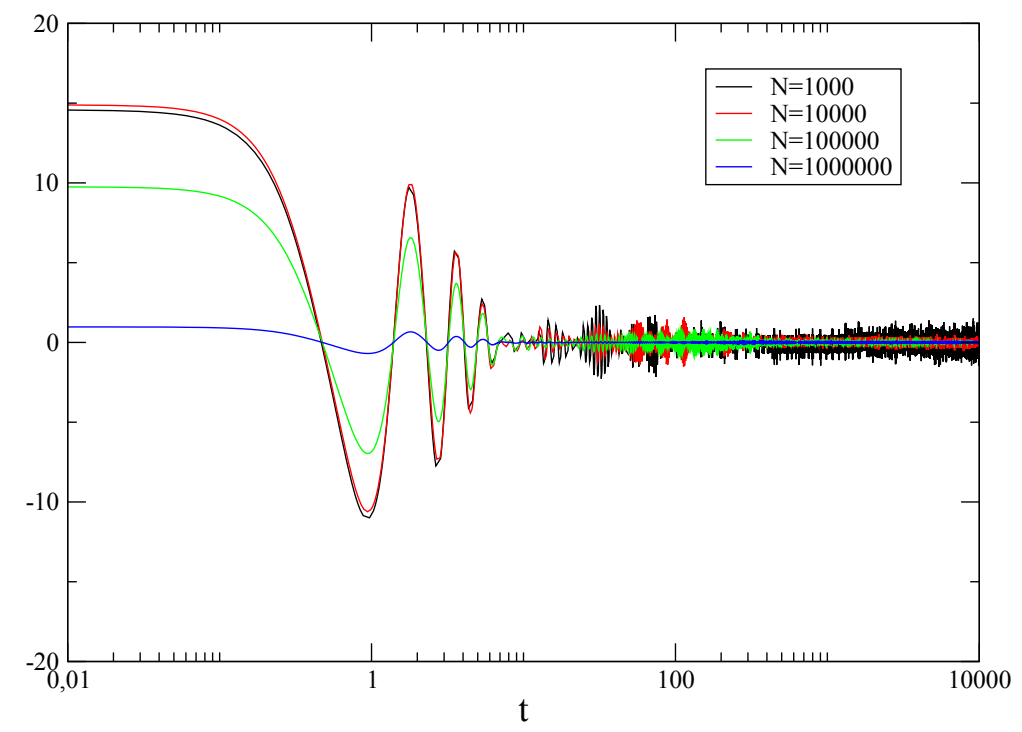

Figura 4.1: Autocorrelação de força $\mathcal{C}(t)$ como função do tempo para diferentes valores de $N$. As condições iniciais são as mesmas do estado homogêneo da Fig. 2.3 termalizada em $t=100.0$ antes de começar as simulações apresentadas.

As Figuras 4.1 e 4.2 retratam $\mathcal{C}(t)$ para diferentes números de partículas 


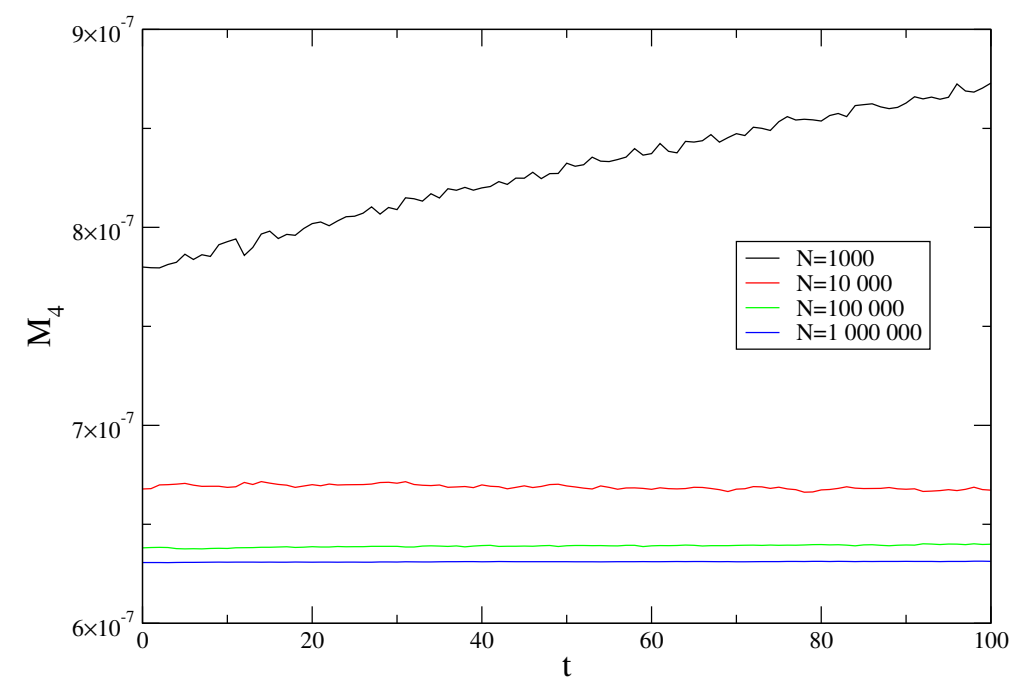

Figura 4.2: Evolução temporal do quarto momento estatístico $\left\langle M_{4}\right\rangle$ da variável $\theta$ média sobre 1000 realizações exceto para $N=100000$ e $N=1000000$ com 300 e 200 realizações, respectivamente. As condições iniciais são as mesmas do estado homogêneo da Fig. 2.3 termalizada em $t=100.0$ antes de começar as simulações apresentadas.

para o modelo estudado e a evolução temporal de $\left\langle M_{4}\right\rangle$ da variável $\theta$. Observamos que o tempo necessário para um decaimento significativo de $\mathcal{C}(t)$, i. e. o tempo de correlação $t_{c}$, é aproximadamente o mesmo para todo os valores de $N$, enquanto o tempo da dinâmica $t_{d}$ é menor para menores valores de $N$ como mostrado na Fig. 4.1. Desta forma, o tempo de correlação pode se tornar da mesma ordem de magnitude que o tempo dinâmico, quebrando a condição de markovianização e, consequentemente, a forma usual de se derivar as equações cinéticas a partir da hierarquia BBGKY não será mais válida. A Figura 4.2 mostra o quarto momento estatístico $M_{4}$ da variável $\theta$ evidenciando que a markovianização não é válida para $N=1000$ e $N=3000$, 
enquanto ela é aproximadamente válida para $N=5000$. Para $N>10000 \mathrm{o}$ sistema é claramente markoviano, em concordância com os resultados da Tabela 2.1. Isso explica porque uma reescala diferente em $N^{\delta}$ na dinâmica com $\delta \neq 2$ é observada em sistemas homogêneos e unidimensionais com pequenos valores de $N[28]$.

\subsection{O Modelo Hamiltoniano de Campo Médio}

O Modelo Hamiltoniano de Campo Médio que foi introduzido por S. Ruffo e M. Antoni [1] (em inglês, chamado de "Hamiltonian Mean FIeld- HMF) é um sistema de partículas de massas iguais se movendo em um círculo, acopladas por um potencial de interação da forma $\pm\left[1-\cos \left(\theta_{i}-\theta_{j}\right)\right]$ (atrativa ou repulsiva para o sinal + ou - , respectivamente), onde $\theta_{i}$ é o ângulo que dá a posição da i-ésima partícula no círculo.

Uma generalização bi-dimensional do modelo HMF foi introduzida por Ruffo e colaboradores na referência [1], mas uma série de artigos sobre este modelo pode ser encontrada nos livros [16, 13, 17].

A Hamiltoniana para o HMF é escrita usualmente na forma [51]

$$
H=\sum_{i=1}^{N} \frac{p_{i}^{2}}{2}+\frac{1}{2 N} \sum_{i, j=1}^{N}\left[1-\cos \left(\theta_{i}-\theta_{j}\right)\right]
$$

em que $\theta_{i} \in\left[-\pi, \pi\left[\right.\right.$ e $p_{i}$ é o momento canonicamente conjugado à $\theta_{i}$. Por 
analogia, definimos a magnetização para os rotores, na forma

$$
\mathbf{M}=\frac{1}{N} \sum_{i=1}^{N}\left(\cos \theta_{i}, \sin \theta_{i}\right)
$$

com componentes $M_{x}=\frac{1}{N} \sum_{i=1}^{N} \cos \theta$ e $M_{y}=\frac{1}{N} \sum_{i=1}^{N} \sin \theta$.

Para obter as propriedades de equilíbrio desse sistema notamos que escolhemos a origem dos ângulos do sistema de modo que $M_{x}=0$. Denotando $M_{y}=M$, podemos escrever o potencial na forma

$$
V_{0}=\frac{1}{2 N}\left(1-M^{2}\right)
$$

Assim, a Hamiltoniana pode ser escrita na forma

$$
H=\sum_{i=1}^{N} \frac{p_{i}^{2}}{2}+\frac{1}{2 N}\left(1-M^{2}\right) .
$$

Os resultados para o equilíbrio no ensemble canônico podem ser obtidos por meio da função-partição:

$$
Z=\int d^{N} p_{i} d^{N} \theta_{i} \exp (-\beta H)
$$

em que $\beta=\left(k_{B} T\right)^{-1}$, e o domínio de integração é estendido à todo o espaço de fase. Substituindo a Hamiltoniana (4.16) na função de partição (4.17) e 
integrando sobre os momentos, obtemos

$$
Z=\left(\frac{2 \pi}{\beta}\right)^{N / 2} \int_{-\pi}^{\pi} d^{N} \theta_{i} \exp \left[\frac{-\beta \varepsilon N}{2}\left(1-M^{2}\right)\right]
$$

Para a integração em $\theta$ utilizamos a transformação de Hubbard-Stratonovich:

$$
\exp \left[\frac{\mu}{2} x^{2}\right]=\frac{1}{\pi} \int_{-\infty}^{\infty} d y \exp \left[-y^{2}+\sqrt{2 \mu} x y\right]
$$

em que $\mu \in \mathbb{R}$. Com isso podemos escrever

$$
Z=\left(\frac{2 \pi}{\beta}\right)^{N / 2} \exp \left[\frac{-\beta \varepsilon N}{2}\right] J
$$

com

$$
J=\frac{1}{\pi} \int_{-\pi}^{\pi} d^{N} \theta_{i} \int_{-\infty}^{\infty} d y \exp \left[-y^{2}+\sqrt{2 \mu} M y\right]
$$

e $\mu=\beta \varepsilon N$. Fazendo a mudança de variável $y \rightarrow y \sqrt{N / 2 \beta \varepsilon}$, temos então a seguinte expressão para $J$ :

$$
J=\frac{N}{2 \pi \beta \varepsilon} \int_{-\infty}^{\infty} d y \exp \left\{-N\left[\frac{y^{2}}{2 \beta \varepsilon}-\ln \left(2 \pi I_{0}(y)\right)\right]\right\}
$$

em que $I_{n}$ é a função de Bessel modificada de ordem $n$. Por fim podemos calcular essa última integral por meio da técnica de ponto de cela no limite de campo médio $(N \rightarrow \infty)$. Neste limite a energia livre de Helmholtz por 
partícula tem a forma

$$
\beta f=\lim _{N \rightarrow \infty} \frac{\ln Z}{N}=-\frac{1}{2}\left(\frac{2 \pi}{\beta}\right)+\frac{\varepsilon \beta}{2}+\max _{y}\left\{\frac{y^{2}}{2 \beta \varepsilon}-\ln \left[2 \pi I_{0}(y)\right]\right\} .
$$

A condição de máximo nos leva à equação de consistência

$$
\frac{y}{\varepsilon \beta}=\frac{I_{1}(y)}{I_{0}(y)}
$$

Se acoplarmos um campo externo $h$ à Hamiltoniana e derivarmos a energia livre em relação a tal campo, para o valor nulo do campo $h=0$ chegamos a:

$$
M=\frac{I_{1}(\bar{y})}{I_{0}(\bar{y})} .
$$

com $\bar{y}$ sendo a solução da equação (4.19). Uma dedução mais simples da distribuição de equilíbrio para esse sistema foi obtido por Rocha-Filho e colaboradores [23].

\subsubsection{A Markovianização para o HMF}

A fim de corroborar nossos resultados e complementar os resultados obtidos por Rocha Filho e colaboradores em 2014, realizamos simulações semelhantes para o HMF utilizando diferentes energias e números de partículas. Utilizamos novamente um Runge-Kutta de quarta ordem nas integrações numéricas [50], com passo de tempo $\delta t=0.1$ e erro máximo aceito de $10^{-5}$. A relação entre o tempo que a correlação de força leva para se anular e a 
dinâmica do sistema durante esse tempo é apresentada nas figuras a seguir, reforçando a tese defendida em nosso estudo anterior.

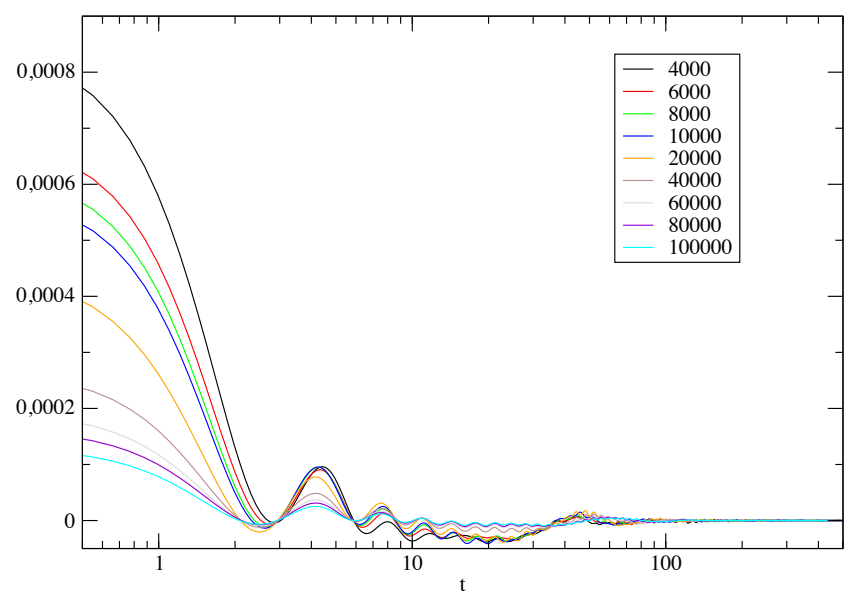

Figura 4.3: Evolução temporal da autocorrelação de força $\mathcal{C}(t)$. Média sobre 1000 realizações para $N=4000$ até $N=80000$ e 300 realizações para $N=$ 100000 partículas, utilizando uma condição inicial de water bag e e=0,69.

A tabela 4.1 apresenta um comparativo entre o expoente que melhor ajusta duas curvas da simulação dinâmica para $M_{4}$ com dois números de partículas diferentes para um sistema com energia 0,69 determinado por meio do Método de Mínimos Quadrados.

A tabela 4.2 apresenta um comparativo entre o expoente que melhor ajusta duas curvas da simulação dinâmica para $M_{4}$ com dois números de partículas diferentes para um sistema com energia 0,8 , contruída da mesma forma que a tabela 4.2 .

Os resultados apresentados nas tabelas 4.1 e 4.2 poderiam ser ainda me- 


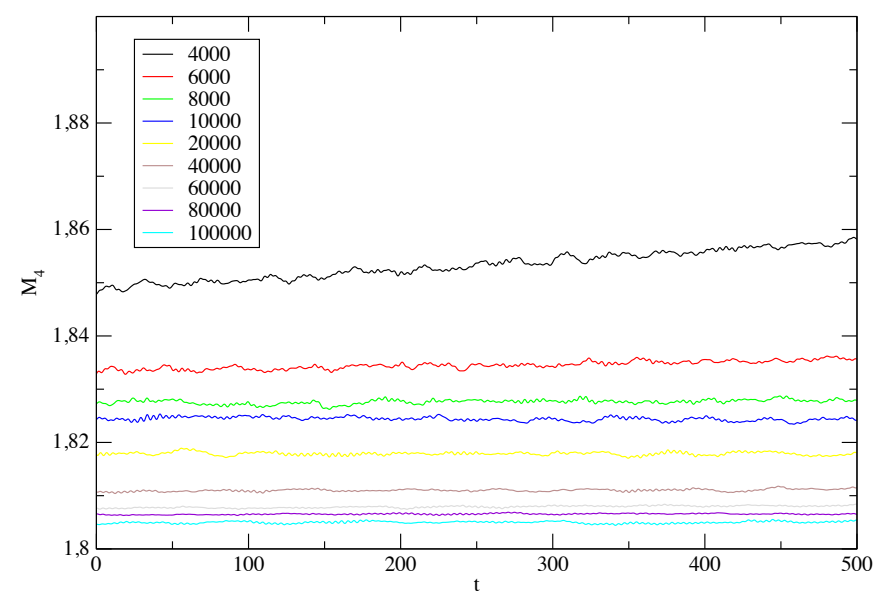

Figura 4.4: Evolução temporal do quarto momento estatístico $\left\langle M_{4}\right\rangle$ para a variável $\theta$. Média sobre 1000 realizaçõespara $N=4000$ até $N=80000$ e 300 realizações para $N=100000$ partículas, utilizando uma condição inicial de water bag e $\mathrm{e}=0,69$.

\begin{tabular}{|c|c|c|}
$N_{1}$ & $N_{2}$ & $\delta$ \\
\hline \hline 300 & 1000 & 1.701 \\
1000 & 3000 & 1.786 \\
3000 & 5000 & 2.103 \\
5000 & 10000 & 2.061 \\
10000 & 20000 & 2.092 \\
20000 & 40000 & 2.046 \\
40000 & 60000 & 2.016
\end{tabular}

Tabela 4.1: Melhor reescala em $N^{\delta}$ para o momento $M_{4}$ entre um par de dados simulados com $N_{1}$ e $N_{2}$ partículas com energia $e=0,69$.

lhores caso o tempo total da simulação fosse maior e o erro máximo admitido fosse inferior a $10^{-4}$ que foi o valor máximo de erro adotado nas simulações.

Note que o expoente próximo de 1.7 só se apresenta em simulações com 


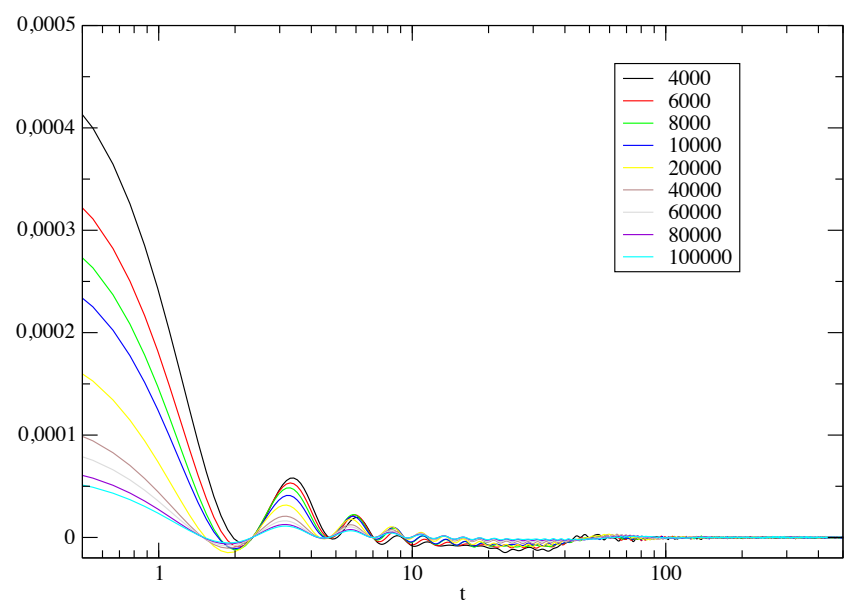

Figura 4.5: Evolução temporal da autocorrelação de força $\mathcal{C}(t)$. Média sobre 1000 realizações para $N=4000$ até $N=80000$ e 300 realizações para $N=$ 100000 partículas, utilizando uma condição inicial de water bag e e=0,8.

\begin{tabular}{|c|c|c|}
$N_{1}$ & $N_{2}$ & $\delta$ \\
\hline \hline 300 & 1000 & 1.856 \\
1000 & 3000 & 1.765 \\
3000 & 5000 & 1.958 \\
5000 & 10000 & 2.066 \\
10000 & 20000 & 2.012 \\
20000 & 40000 & 2.001 \\
40000 & 60000 & 2.075
\end{tabular}

Tabela 4.2: Melhor reescala em $N^{\delta}$ para o momento $M_{4}$ entre um par de dados simulados com $N_{1}$ e $N_{2}$ partículas com energia $e=0,8$.

$N$ pequeno. Na medida em que $N \rightarrow \infty$, o melhor ajuste se dá próximo do expoente 2 . 


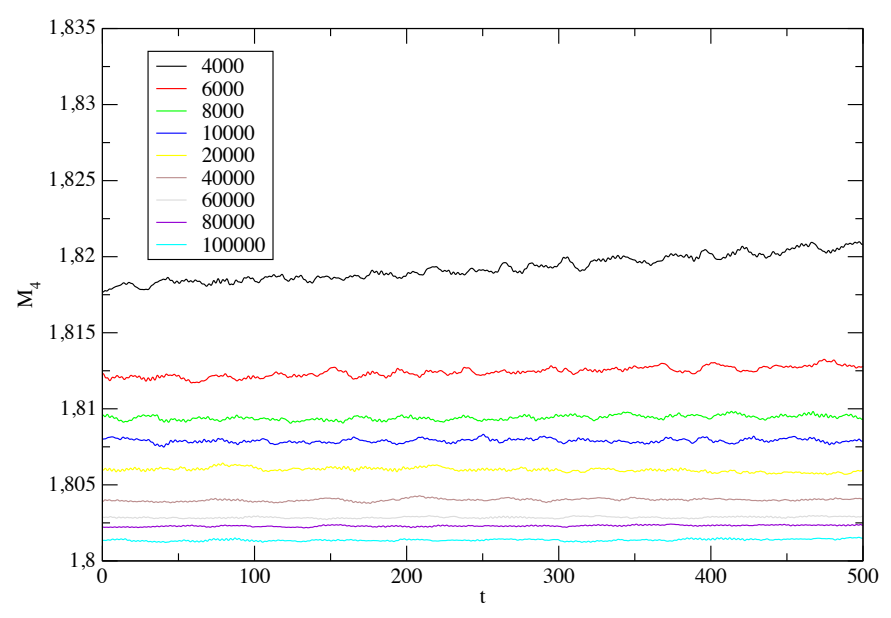

Figura 4.6: Evolução temporal do quarto momento estatístico $\left\langle M_{4}\right\rangle$ para a variável $\theta$. Média sobre 1000 realizações para $N=4000$ até $N=80000$ e 300 realizações para $N=100000$ partículas, utilizando uma condição inicial de water bag e $\mathrm{e}=0,80$.

\subsection{O Modelo do Anel}

Introduzido em 2001, por Sota, Iguchi, Morikawa,Tatekawa e Maeda, o Modelo do anel (em inglês chamado de "ring-model") é um modelo simplificado de um sistema auto-gravitante. Neste modelo, $N$ partículas de massa iguais a 1 se deslocam em um círculo de raio unitário interagindo mutuamente por meio de forças gravitacionais [43]. O potencial e a Hamiltoniana são da forma

$$
V_{i j}=\frac{1}{2 N \sqrt{2}} \sum_{i<j=1}^{N} \frac{1}{\sqrt{1-\cos \left(\theta_{i}-\theta_{j}\right)+\varepsilon}}
$$


e

$$
H=\sum_{i=1}^{N} \frac{p_{i}^{2}}{2 m_{i}}+\frac{1}{2 N \sqrt{2}} \sum_{i<j=1}^{N} \frac{1}{\sqrt{1-\cos \left(\theta_{i}-\theta_{j}\right)+\varepsilon}} .
$$

em que $\theta_{i}$ e $\theta_{j}$ estão compreendidos no intervalo $-\pi<\theta<\pi$ e representam as posições angulares ocupadas pela i-ésima partícula no anel e $\varepsilon$ é um parâmetro de amortecimento. Veja a ilustração 4.7

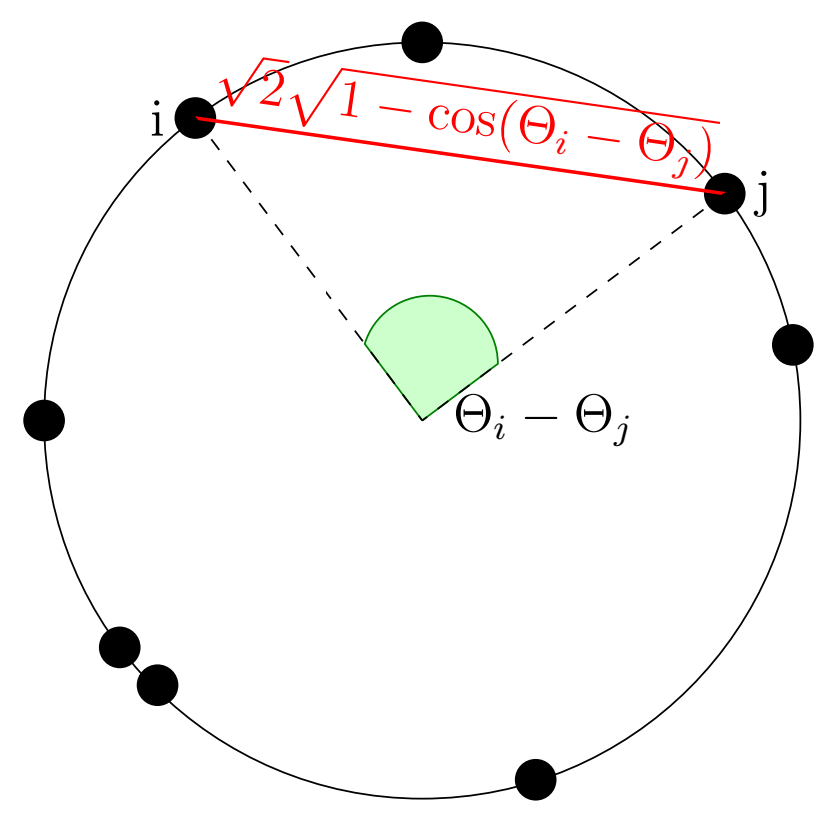

Figura 4.7: $\theta_{i}$ e $\theta_{j}$ estão compreendidos no intervalo $-\pi<\theta<\pi$ e representam as posições angulares ocupadas pela i-ésima partícula no anel.

\subsubsection{A Markovianização para o Modelo do Anel}

Repetimos a análise proposta anteriormente para o HMF. Realizamos simulações semelhantes utilizando diferentes energias e números de partículas. A relação entre o tempo que a correlação de força leva para se anular e a 
dinâmica do sistema durante esse tempo é a que segue nos gráficos 4.2.1 e 4.2.1, para os quais foi utilizado um waterbag com energia 0, 34 e passo no tempo de 0,03 utilizando um Runge-Kutta de quarta ordem na integração numérica [50]. O erro máximo admitido foi de $10^{-6}$ e o $\varepsilon$ utilizado foi de 0,01. Note que as simulações para o Ring-model apresentam muito mais ruído que as do HMF. Além disso, são simulações muito mais onerosas o que significa que levam muito mais tempo para serem concluídas (o tempo de simulação escala com $N^{2}$ para este modelo), motivo pelo qual apresentamos menos números de partículas neste sistema.

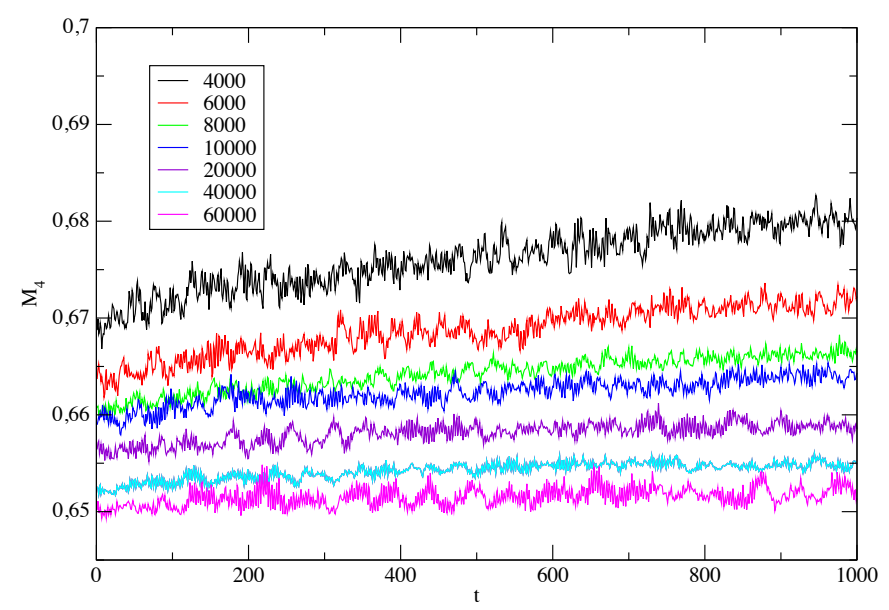

Figura 4.8: Evolução temporal do quarto momento estatístico $\left\langle M_{4}\right\rangle$ para a variável $\theta$. Média sobre 50 realizações para $N=4000$ até $N=60000$ e 5 realizações para os demais números de partículas, utilizando uma condição inicial de waterbag e $e=0,34$ termalizado em 100 .

Mais uma vez, a tabela a seguir apresenta um comparativo entre o expo- 


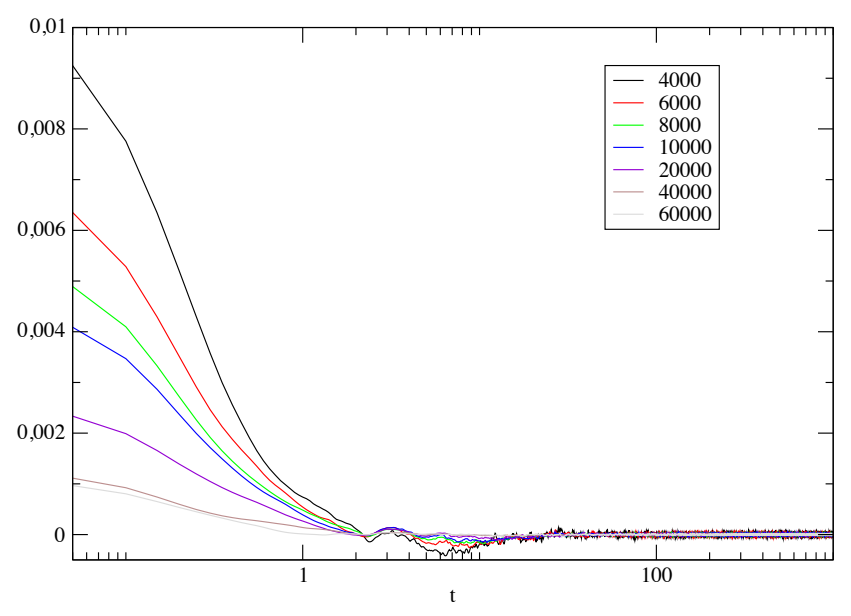

Figura 4.9: Evolução temporal da autocorrelação de força $\mathcal{C}(t)$. Média sobre 50 realizações para $N=4000$ até $N=60000$ e 5 realizações para os demais números de partículas, utilizando uma condição inicial de waterbag e $e=0,34$ termalizado em 100.

ente que melhor ajusta duas curvas da simulação dinâmica para $\left\langle M_{4}\right\rangle$ com alguns números de partículas diferentes.

\begin{tabular}{|c|c|c|}
$N_{1}$ & $N_{2}$ & $\delta$ \\
\hline \hline 300 & 1000 & 1.743 \\
1000 & 3000 & 1.910 \\
3000 & 5000 & 1.993 \\
5000 & 10000 & 2.084 \\
10000 & 20000 & 1.998
\end{tabular}

Tabela 4.3: Melhor reescala em $N^{\delta}$ para o momento $M_{4}$ entre um par de dados simulados com $N_{1}$ e $N_{2}$ partículas sem termalização.

O expoente próximo de 1.7 só se apresenta em simulações com $N$ pequeno. Na medida em que $N \rightarrow \infty$, o melhor ajuste se dá próximo do expoente 2, 
corroborando os resultados obtidos para os demais modelos. 


\section{Capítulo 5}

\section{Conclusões e Perspectivas}

Neste trabalho, mostramos que o modelo de Spins Anisotrópicos de Heisenberg introduzido por Gupta e Mukamel em [30] é efetivamente um sistema hamiltoniano clássico e unidimensional. Estudamos seu equilíbrio pela maximização da entropia. Provamos também que a dinâmica de um QSS escala com $N^{2}$ para grandes valores de $N$ enquanto a escala relatada anteriormente de $N^{1.7}$ deve-se a alguns efeitos não-markovianos da dinâmica. Para $N$ grande, as equações cinéticas de um sistema com interações de longoalcance homogêneo e unidimensional devem levar em consideração as colisões entre 3 partículas que são de ordem $1 / N^{2}$. Essa abordagem só é válida para $N$ suficientemente grande tal que o termo de colisão entre duas partículas efetivamante se anula. O caso em que $N$ é pequeno (não tão grande) pode ser descrito pela abordagem desenvolvida por Ettoumi e Firpo para a determinação de coeficientes de difusão em termos das variáveis de ação. Eles 
utilizaram uma abordagem de tempo médio e obtiveram um escalonamento (reescala) $N^{1.7}$ para o HMF.

Além do estudo para o modelo de Spins Anisotrópicos, verificamos e comprovamos nossa hipótese por meio de simulações com modelos conhecidos (HMF - $e=0.69$ e $e=0.80$ - e Ring Model).

O desenvolvimento deste trabalho, nos indicou várias outras análises possíveis que servirão de perspectivas para trabalhos futuros, como

- Calcular o termo de segunda ordem que domina a equação cinética para $\mathrm{N}$ grande partindo das equações de Landau e Balescu-Lenard;

- Com base na evolução temporal da auto-correlação de força, avaliar o comportamento de sistemas não-homogêneos e unidimensionais assim como para sistemas de dimensão maior para o caso homogêneo. Definir se isso influencia (ou de que forma) a reescala para números de partículas pequenos;

- Estudar o modelo mais recente introduzido por Gupta e Mukamel de spins clássicos em uma esfera descrito por uma Hamiltoniana bidimensional que também exibie um escalonamento da dinâmica de um QSS com $N^{1.7}$. Avaliar se esse sistema não é de fato unidimensional e não bidimensional como se defende em [31]. 


\section{Apêndice A - Os momentos}

\section{Estatísticos}

O momento de ordem $n$ de uma função densidade de probabilidade é definido por

$$
\left\langle x^{n}\right\rangle=\int_{-\infty}^{\infty} x^{n} P_{X} d x .
$$

Os momentos de uma distribuição são os que seguem:

- Primeiro Momento - Média

O primeiro momento é o valor médio dos valores do conjunto $S_{X}$, ou seja, a média ponderada de todos valores com o peso dado pelas respectivas probabilidades.

$$
\langle x\rangle=\int_{-\infty}^{\infty} x P_{X} d x .
$$

Em nosso estudo do sistema homogêneo, este momento para a variável $\theta$ se anula. 
- Segundo Momento - Variância

O segundo momento mede a dispersão, ou seja, o quanto a função densidade de probabilidades está deslocada em relação à média.

$$
\left\langle x^{2}\right\rangle=\int_{-\infty}^{\infty} x^{2} P_{X} d x
$$

Também pode ser chamado de variância e definido pelo valor médio do quadrado do desvio em relação à média.

$$
\left\langle(x-\langle x\rangle)^{2}\right\rangle=\int_{-\infty}^{\infty}(x-\langle x\rangle)^{2} P_{X} d x .
$$

Fisicamente, o segundo momento para a variável $\theta$ representa a energia cinética que é praticamente constante em nossos sistemas.

- Terceiro Momento

$$
\left\langle x^{3}\right\rangle=\int_{-\infty}^{\infty} x^{3} P_{X} d x
$$

O terceiro momento está relacionado com a assimetria da função densidade de probabilidades ou obliquidade (skewness em inglês $\gamma)$ definida por

$$
\gamma=\left\langle\frac{(x-\langle x\rangle)^{3}}{\sigma}\right\rangle
$$

A skewness mede o quanto a função densidade de probabilidades está destorcida em relação a sua simetria em torno da média. O 
terceiro momento para a variável $\theta$ também se anula em nossos sistemas de estudo.

- Quarto Momento

$$
\left\langle x^{4}\right\rangle=\int_{-\infty}^{\infty} x^{4} P_{X} d x
$$

O quarto momento está relacionado com a curtose $(\kappa)$ ou kurtosis, em inglês, da função densidade de probabilidades

$$
\kappa=\frac{\langle x\rangle^{4}}{\left\langle x^{2}\right\rangle^{2}}-3 .
$$

A curtose é uma medida comparativa que fornece o achatamento ou agudeza da função densidade de probabilidades em relação à distribuição normal ou gaussiana. Em nosso estudo, avaliamos o excesso de curtose para a variável $\theta$ na dinâmica do sistema rumo ao equilíbrio, assim, espera-se um valor que se aproxime de 3 com o passar do tempo.

- Quinto Momento

$$
\left\langle x^{5}\right\rangle=\int_{-\infty}^{\infty} x^{5} P_{X} d x .
$$

O quinto momento, como todos os de ordem ímpar em distribuições pares como as distribuições de velocidade estudadas neste trabalho, se anula. 
- Sexto Momento

$$
\left\langle x^{6}\right\rangle=\int_{-\infty}^{\infty} x^{6} P_{X} d x
$$

O sexto momento estatístico das velocidades é apresentado em nosso trabalho apenas como uma forma de corroborar a reescala adequada para a dependência evidenciada entre o número de partículas do sistema e seu tempo de permanência no estado quasiestacionário. 


\section{Apêndice B - Método dos Quadrados Mínimos}

Reproduzimos a seguir o algoritmo em $C$ utilizado para montar as tabelas que apresentam os expoentes relacionados aos números de partículas em pares de simulações para o estudo sobre o spin anisotrópico de campo médio. Uma adaptação deste programa foi utilizada para construir as tabelas para os demais modelos estudados. Os textos em itálico precedidos por um $c$ são meus comentários sobre etapas do programa:

\#INCLUDE $<$ STDLIB.H $>$

\#INCLUDE $<$ MATH.H $>$

\#INCLUDE < STDIO.H $>$

\#INCLUDE "MINIMIZAR.INC" 
DOUBLE SCALING( INT, INT, INT, INT, DOUBLE, DOUBLE *, DOUBLE *, DOUBLE *, DOUBLE *);

$\operatorname{INT} \operatorname{MAIN}()$

\{

INT I,N1,N2,NPOINTS1,NPOINTS2,NSCL;

DOUBLE SCAL_MIN,SCAL_MAX,PR,ERRO,SCL,DSCL,EMIN,SCM; DOUBLE TIME1[DIMMAX],TIME2[DIMMAX],MOM1[DIMMAX], MOM2[DIMMAX];

FILE $*$ IN,$*$ ENTRADA $1,{ }^{*}$ ENTRADA2,${ }^{*}$ SAIDA;

c Lê alguns parâmetros de entrada $\mathrm{IN}=\mathrm{FOPEN}(" \mathrm{MINIMIZAR}$.IN","R"); SAIDA=FOPEN("MINIMIZAR.DAT","W");

c Número de partículas utilizadas na primeira $N_{1}$ e na segunda $N_{2}$ simulações

FSCANF(IN,"\%I",\&N1);

FSCANF(IN,"\%I",\&N2);

c Número de linhas do primeiro e do segundo arquivo dat que contêm os momentos considerando-se o arquivo com menos linhas FSCANF(IN,"\%I",\&NPOINTS1); FSCANF(IN,"\%I",\&NPOINTS2);

c Valores mínimo e máximo de teste para expoente de ajuste das curvas FSCANF(IN,"\%LF",\&SCAL_MIN); 
FSCANF(IN,"\%LF",\&SCAL_MAX);

c Número de pontos considerados para se calcular os mínimos quadrados

FSCANF(IN,"\%I",\&NSCL);

FCLOSE(IN);

c abre os arquivos dat de onde serão lidos os pontos (tempo,momento) para o cálculo do ajuste ENTRADA1=FOPEN("MOMS1.DAT","R"); ENTRADA2=FOPEN("MOMS2.DAT","R");

c Lê os valores de tempo, posição e quarto momento estatístico gravados nos arquivos moms1.dat e moms2.dat até a linha definida por npoints* no minimizar.in FOR $(\mathrm{I}=0 ; \mathrm{I}<\mathrm{NPOINTS} 1 ; \mathrm{I}++)$

\{ FSCANF(ENTRADA1, "\%LF \%LF \%LF",\&TIME1[I],\&PR,\&MOM1[I]); \}

FOR $(\mathrm{I}=0 ; \mathrm{I}<\mathrm{NPOINTS} 2 ; \mathrm{I}++)$

\{

FSCANF(ENTRADA2, "\%LF \%LF \%LF" ,\&TIME2[I],\&PR,\&MOM2[I]); 
Segue, também, o algoritmo do SCALING.C chamado dentro do processo MINIMIZAR.C:

$$
\begin{aligned}
& \text { \#INCLUDE }<\text { STDLIB.H }> \\
& \text { \#INCLUDE }<\text { MATH.H }> \\
& \text { \#INCLUDE }<\text { STDIO.H }>
\end{aligned}
$$

\#INCLUDE "MINIMIZAR.INC"

DOUBLE SCALING( INT NPOINTS1, INT NPOINTS2, INT N1, INT N2, DOUBLE SC, DOUBLE *TIME1, DOUBLE *TIME2, DOUBLE *MOM1, DOUBLE *MOM2)

INT I,K,MM;

DOUBLE ERRO,T1MAX,T2MAX,TA,TB,MA,MB,MINT,TT1[DIMMAX], TT2[DIMMAX];

c Para os valores de tempo lidos, calcula o tempo dividido pelo número de partículas correspondente elevado a um certo expoente SC compreendido entre os dois (mínimo e máximo) lidos em minimizar.in FOR $(\mathrm{I}=0 ; \mathrm{I}<$ NPOINTS1; +++$)$ 
TT1[I]=TIME1[I]/POW((DOUBLE) N1,SC);

\}

FOR $(\mathrm{I}=0 ; \mathrm{I}<\mathrm{NPOINTS} 2 ; \mathrm{I}++)$

\{

TT2[I]=TIME2[I]/POW((DOUBLE) N2,SC);

\}

c calcula o erro entre os novos valores de momento para os dois números de partículas e diferentes expoentes e escolhe o expoente que apresenta menor erro, ou seja, melhor ajuste

T1MAX $=$ TT1[NPOINTS1-1];

$\mathrm{T} 2 \mathrm{MAX}=\mathrm{TT} 2[\mathrm{NPOINTS2}-1]$

$\mathrm{K}=1$;

$\mathrm{ERRO}=0.0$

$\mathrm{MM}=0$

FOR $(\mathrm{I}=1 ; \mathrm{I}<\mathrm{NPOINTS} 2 ; \mathrm{I}++)$

\{

IF $(\mathrm{TT} 2[\mathrm{I}]<\mathrm{T} 1 \mathrm{MAX})$

\{ 


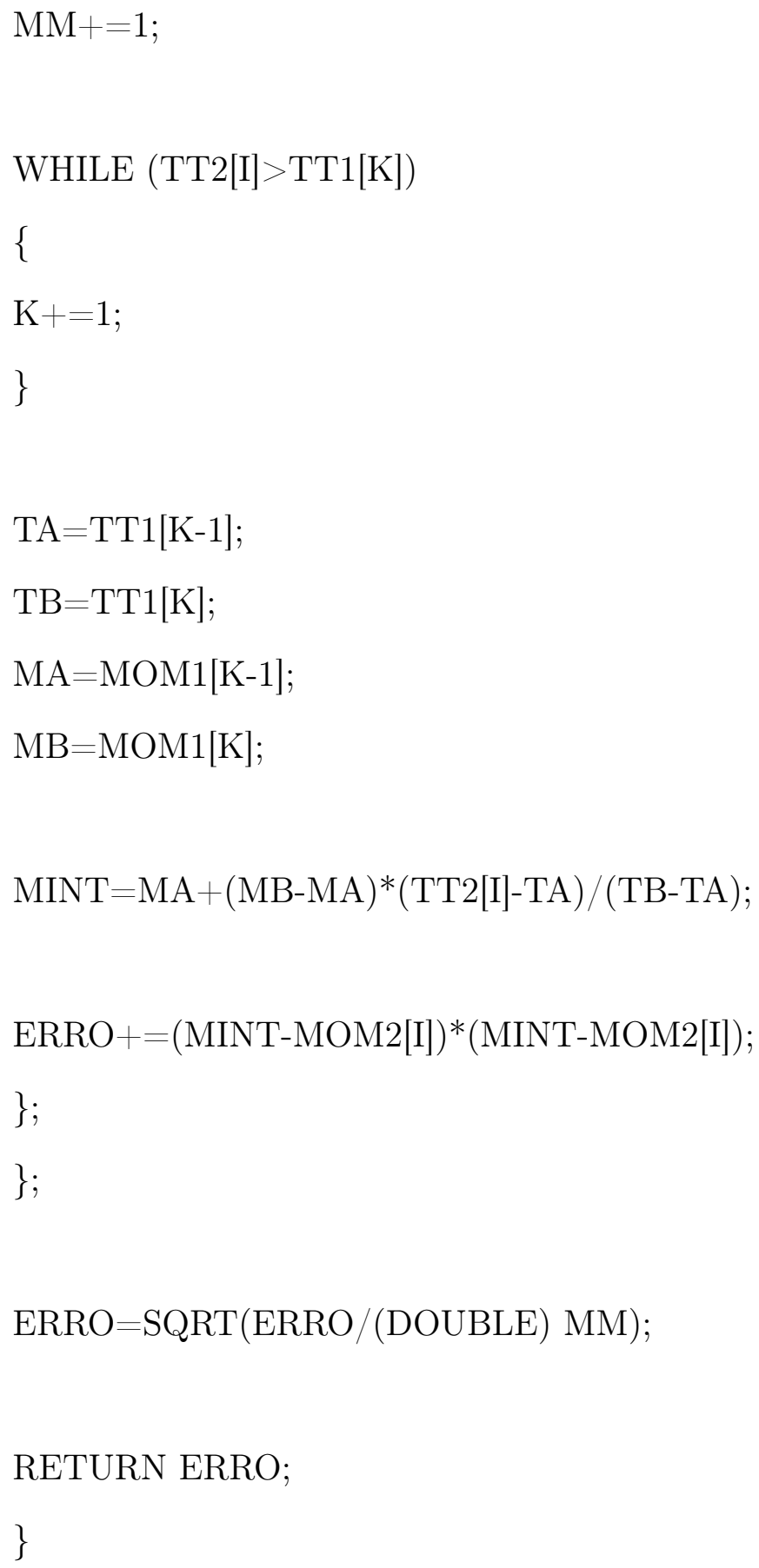




\section{Referências Bibliográficas}

[1] M. Antoni e S. Ruffo. Clustering and relaxation in hamiltonian long-range dynamics. Phys. Rev. E, 52(2361), 1995.

[2] F. Antunes. Relaxação ao equilíbrio termodinâmico em sistemas com interações de longo alcance. Dissertaçao de Mest., Universidade Federal do Rio Grande do Sul, 2014.

[3] I. Arad e D. Lynden Bell. Inconsistency in relaxation theories. Mon. Not. R. Astr., 361:385, 2005.

[4] M. Badino. The foundational role of ergodicity theory. Found. of Sc., 11:323, 2006.

[5] R. Balescu. Irreversible processes in ionized gases. Phys. Fluids, 3:52, 1960 .

[6] R. Balescu. Statistical Dynamics - Matter out of Equilibrium. Imperial College Press, 1997. 
[7] J. Barré, D. Mukamell, e S. Ruffo. Inequivalence of ensembles in a system with long range interactions. Phys. Rev. Let., 87(030601), 2001.

[8] D. Lynden Bell. Statistical mechanics of violent relaxation in stellar systems. Mon. Not. R, Astr. Soc., 36:101, 1967.

[9] G. Bertin e M. Trenti. Thermodynamical description of a family of partially relaxed stellar systems. Astro. Phys. Journal, 584:729, 2003.

[10] J. Binney e S. Tremaine. Galactic Dynamics. Princeton University Press, USA, 1987.

[11] M.N. Bogoliubov. Kinetic equations. J. Phys. (USSR), 10(265), 1946.

[12] A. Campa, T. Dauxois, e S. Ruffo. Statistical mechanics and dynamics of solvable models with long-range interactions. Phy. Rep., 480:57, 2009.

[13] A. Campa, A. Giansanti, G. Morigi, e F. S. Labini, editors. Dynamics and Thermodynamics with Long-Range Interactions: Theory and Experiments, volume 970. AIP Conf. Proceedings, 2008.

[14] P.H. Chavanis. Kinetic equations for systems with long-range interactions: a unified description. J. Stat. Mech., (05019), 2010.

[15] M. D'Agostino, F. Gulminelli, Ph. Chomaz, M. Bruno, F. Cannata, R. Bougault, N. Colonna, F. Gramegna, I. Iori, N. Le Nein- 
dre, G. V. Margagliotti, P. F. Mastinu, P. M. Milazzo, A. Moroni, $e$ G. Vannini. Negative heat capacity in the critical region of nuclear fragmentation: an experimental evidence of the liquid-gas phase transition. Phys. Let. B, 473:219, 2000.

[16] T. Dauxois, S. Ruffo, E. Arimondo, e M.Wilkens. Dynamics and Thermodynamics of Systems with Long Range Interactions: An Introduction. Springer, 2002.

[17] T. Dauxois, S. Ruffo, e L. F. Cugliandolo. Long-Range Interacting Systems. Oxford Univ. Press, 2010.

[18] W. Ettoumi e M.C. Firpo. Action diffusion and lifetimes of quasistationary states in the hamiltonian mean-field model. Phys. Rev. E, 87(030102 (R)), 2013.

[19] A. Figueiredo, T. M. Rocha Filho, e M. A. Amato. Ergodicity and central-limit theorem in systems with long-range interactions. EPL, 83(30011), 2008.

[20] A. Figueiredo, T. M. Rocha Filho, M. A. Amato, Z. T. Oliveira Jr, e R. Matsushita. Truncated lévy flights and weak ergodicity breaking in the hamiltonian mean field model. Phys. Rev. E, 89(022106), 2014.

[21] T. M. Rocha Filho. Solving the vlasov equation for onedimensional models with long range interactions on a gpu. Comp. Phys. Com., 184:34, 2013. 
[22] T. M. Rocha Filho. Molecular dynamics for long-range interacting systems on graphic processing units. Comp. Phys. Com., 185:1364, 2014.

[23] T. M. Rocha Filho, M. A. Amato, e A. Figueiredo. A novel approach for the determination of equilibrium properties of classical hamiltonian systems with long-range interactions. J. Phys. A, 42(16), 2009.

[24] T. M. Rocha Filho, M. A. Amato, e A. Figueiredo. Nonequilibrium phase transitions and violent relaxation in the hamiltonian mean-field model. Physical Review. E, 85(062103), 2012.

[25] T. M. Rocha Filho e A. Figueiredo. Maple package for the symmetry analysis of differential equations. Comp. Phys. Com., 182:467, 2011.

[26] T. M. Rocha Filho, A. Figueiredo, e M. A. Amato. Entropy of classical systems with long-range interactions. Phys. Rev. Let., 95(1906011), 2005.

[27] T. M. Rocha Filho, B. A. Mello, A. Figueiredo, e M. A. Amato. Phase transitions in simplified models with long-range interactions. Phys. Rev. E, 84(041121-1), 2011.

[28] T. M. Rocha Filho, A. E. Santana, M. A. Amato, e A. Figueiredo. Scaling of the dynamics of homogeneous states of 
one-dimensional long-range interacting systems. Phys. Rev. E, 90(032133), 2014.

[29] J. W. Gibbs. Elementary Principles in Statistical Mechanics with Especial Reference to the Rational Foundation of Thermodynamics. Yale University Press, 1902.

[30] S. Gupta e D. Mukamel. Quasistationarity in a model of classical spins with long-range interactions. Mon. Not. R, Astr., 136:101, 2011.

[31] S. Gupta e D. Mukamel. Quasistationarity in a model of longrange interacting particles moving on a sphere. Phys. Rev. E, 88(052137), 2013.

[32] S.W. Hawking. Black hole explosions? Nature, 248:30, 1974.

[33] L. Hernquist e D.N. Spergel. Formation of shells in major mergers. Astro. Phys. Journal, 399:117, 1992.

[34] P. Hertel $e$ W. Thirring. A soluble model for a system with negative specific heat. Ann. of Phys., 63:520, 1971.

[35] H.Touchette. Ensemble equivalence for general many body systems. EPL, 96(5), 2011.

[36] H.Touchette, R. S. Ellis, e B. Turkington. An introduction to the thermodynamic and macrostate levels of nonequivalent ensembles. Phys. A, 340:138, 2004. 
[37] A. Kull, R.A. Treumann, e H. Böhringer. A note on the statistical mechanics of violent relaxation of phase space elements of different densities. Astro. Phys. Journal, 484:58, 1997.

[38] A. Lenard. On bogoliubov's kinetic equation for a spatially homogeneous plasma. Ann. of Phys., 10:390, 1960.

[39] R. L. Liboff. Kinetic Theory - Classical, Quantum and Relativistic Descriptions. Springer, 2003.

[40] C. R. Lourenço. Processos estocásticos e interações de longo alcance. Dissertaçao de Mest., Universidade de Brasília, 2007.

[41] C. R. Lourenço e T. M. Rocha Filho. Scaling of the dynamics of a homogeneous one-dimensional anisotropic classical heisenberg model with long-range interactions. Phys. Rev. E, 92(012117), 2015.

[42] D. Lynden-Bell e R. M. Lynden-Bell. On the negative specific heat paradox. Mon. Not. R. Astr., 181(405), 1977.

[43] J. Maciel. Estudo de Modelos Simplificados com Interações de Longo Alcance no Ensemble Microcanônico. Tese de Dout. , Universidade de Brasília, 2015.

[44] A. Maritan, M. Cieplak, M. Swift, F. Toigo, e J. Banavar. Random anisotropy blume-emery-griffiths model. Phys. Rev. Let., 69:221, 1992. 
[45] J.C Maxwell. On Boltzmann's Theorem on the average distribution of energy in a system with material points. Cambridge Philosophical Society's Trans., London, 1876.

[46] J. R. Moura. Contribuições à Mecânica Estatística de sistemas com interação de longo alcance. Tese de Dout., Universidade de Brasília, 2014.

[47] J. R. S. Moura, T. M. Rocha Filho, M. A. Amato, A. E. Santana, $e$ A. Figueiredo. Dynamics and physical interpretation of quasistationary states in systems with long-range interactions. Phys. Rev. E, 89(032116), 2014.

[48] Dwight R. Nicholson. Introduction to Plasma Theory. John Wiley and Sons, Inc, 1983.

[49] N.Rostoker e M.N. Rosenbluth. Test particles in a completely ionized plasma. Phys. Fluids, 3:1, 1960.

[50] W. H. Press, S. A. Teukolsky, W. T. Vetterling, e B. P. Flannery. Numerical Recipes. Cambridge University Press, USA.

[51] B. V. Ribeiro. Abordagens Dinâmica e Estocástica na Descrição de Processos Físicos na Física de Plasmas. Tesè de Dout., Universidade de Brasília, 2014.

[52] M. M. Sano. Zero-collision term problem in kinetic theory of onedimensional systems. J. Phys. Soc. Japan, 81(024008), 2012. 
[53] F.H. Shu. On the statistical mechanics of violent relaxation. Astro. Phys. Journal, 225:83, 1978.

[54] C.H.C. Silvestre. Ergodicidade em sistemas autogravitantes em duas e três dimensões, 2012.

[55] L. Souza. Um estudo de escala da dinâmica de estados homogêneos do sistema gravitacional unidimensional um estudo de escala da dinâmica de estados homogêneos do sistema gravitacional unidimensional. Dissertaçao de Mest., Universidade de Brasília, 2015.

[56] J. R. Steiner. Contribuições à Mecânica Estatística de sistemas com interação de longo alcance. Tese de Dout., Universidade de Brasília.

[57] M. Stiavelli e G. Bertin. Statistical mechanics and equilibrium sequences of ellipticals. Mon. Not. R. Astr., 229:61, 1987.

[58] D. Szasz. Boltzmann's ergodic hypotesis, a conjecture for centuries? Springer, 1994.

[59] W.E. Thirring. Systems with negative specif heat. Z.f. Phys., 235:339, 1970. 